\title{
杯芳烃促进的过渡金属催化反应
}

\author{
马志艳 ${ }^{\dagger}$ 李云剑 孙小强杨 科* 李正义* \\ (常州大学石油化工学院 江苏省绿色催化材料与技术重点实验室 江苏常州 213100)
}

\begin{abstract}
摘要 近年来, 过渡金属催化反应已经成为有机化学中构建碳碳键和碳杂原子键的最有效方法之一, 并引起了化学家 的极大研究兴趣. 杯芳烃是继冠醚和环糊精之后的第三代超分子主体化合物, 其配位性能一直是超分子化学研究的热 点. 通过对其下缘的酚羟基、上缘的苯环对位以及连接苯环单元的亚甲基进行设计改造, 杯芳烃可以有效地作为过渡 金属催化剂的配体, 或者与过渡金属正离子组装成为新的过渡金属催化剂, 从而高效地促进反应的进行. 主要介绍了 近十年来，杯芳烃作为不同过渡金属催化剂的配体并用于各种过渡金属催化反应的研究进展.
\end{abstract}

关键词 杯芳烃; 过渡金属催化; 配体; 有机合成

\section{Calixarene Promoted Transition-Metal-Catalyzed Reactions}

\author{
Ma, Zhiyan ${ }^{\dagger} \quad$ Li, Yunjian $^{\dagger} \quad$ Sun, Xiao-Qiang $\quad$ Yang, Ke* Li, Zheng-Yi* \\ (Jiangsu Key Laboratory of Advanced Catalytic Materials and Technology, School of Petrochemical Engineering, \\ Changzhou University, Changzhou, Jiangsu 213100)
}

\begin{abstract}
In recent years, transition metal catalyzed reactions have become one of the most effective methods to construct carbon-carbon bonds and carbon-heteroatom bonds in organic chemistry, and have attracted great research interest among chemists. Calixarene is the third-generation supramolecular host compound after crown ether and cyclodextrin, and its coordination properties have always been a hot spot in supramolecular chemistry. By designing and modifying its phenolic hydroxyl group at the lower edge, the para-position of the benzene ring at the upper edge, and the methylene group connecting the benzene ring unit, calixarene can be used as a transition metal catalyst ligand or assembled with transition metal to form a novel catalyst, both of which can be effectively used to promote the transition metal catalyzed reaction. The research progress of calixarene as a ligand for different transition-metal catalysts and their application in various transition metal catalytic reactions in the past ten years is introduced.
\end{abstract}

Keywords calixarene; transition-metal catalysis; ligand; organic synthesis

近年来，过渡金属催化反应已经发展成为构建有机 化合物 $\mathrm{C}-\mathrm{C}$ 键和 $\mathrm{C}$ 一杂原子键最为有效方法之一[1-4]. 过渡金属催化反应不仅丰富了有机反应的类型，更提高 了有机合成的效率. 在传统的有机合成反应中, 通过取 代反应使惰性的 $\mathrm{C}-\mathrm{H}$ 键直接转化成 $\mathrm{C}-\mathrm{C}$ 键, 该反应 过程是很难实现的, 但是通过使用过渡金属催化反应就 可以容易地实现该转化过程 ${ }^{[5]}$. 此外, 对于传统的傅克 反应，其本身的选择性比较差，但通过使用过渡金属催 化剂，可以高选择性地实现邻位或者间位 $\mathrm{C}-\mathrm{H}$ 键烷基 化反应 ${ }^{[6]}$. 因此, 探索新颖的过渡金属催化反应已引起
有机化学科研工作者们的广泛关注.

超分子化学是一门融合了近代化学、材料科学和生 命科学的新兴学科, 致力于研究通过多个分子组装而成 的功能化体系 ${ }^{[7-8]}$, 它是分子层次以上的化学, 其主要研 究非共价键分子间的弱相互作用(包括氢键、金属配位 作用、亲-疏水相互作用、范德瓦尔斯力、 $\pi-\pi$ 相互作用 和静电效应等). 目前而言, 超分子领域已经被定义和 概念化成一个结构严谨的体系，其基础涉及相当广泛， 分别包括有机化学及构造分子的合成路线、配位化学及 金属离子-配体复合物、物理化学及其相关研究等. 超分

\footnotetext{
* Corresponding authors. E-mail: keyang@cczu.edu.cn; zyli@cczu.edu.cn Received December 22, 2020; revised February 4, 2021; published online February 26, 2021.

Project supported by the National Natural Science Foundation of China (Nos. 21572026, 21702019), the Postgraduate Research \& Practice Innovation Program of Jiangsu Province (No. KYCX20_2525) and the Advanced Catalysis and Green Manufacturing Collaborative Innovation Center of Jiangsu Province. 国家自然科学基金(Nos. 21572026, 21702019)、江苏省研究生科研与实践创新计划(KYCX20_2525)和江苏省先进催化与绿色制造协同创新中心资助项 目.

$\dagger$ 共同第一作者(These authors contributed equally to this work).
} 
子化学与催化反应的交叉结合, 催生了 “超分子催化” 这一前沿热点研究领域. 超分子催化, 因其高催化选择 性和特殊反应性, 受到科研工作者的广泛关注, 它是利 用分子间弱相互作用力调控化学反应方向和速率的催 化反应, 其催化过程是由反应物之一(作为客体) 被主体 识别后形成超分子过渡状态, 从而有选择地与另一反应 分子结合 ${ }^{[9]}$.

最近, 利用离子键、氢键以及动态共价键等分子间 弱相互作用力实现的过渡金属催化反应已经被报道, 而 利用这些分子间弱作用力实现特定位点的过渡金属催 化反应被定义分子为瞬态导向基策略 ${ }^{[10]}$. 这一策略能 使用催化量的瞬态配体以暂时和可逆的方式将特定基 团转变为导向基团, 以进行金属催化反应, 而在催化完 成后不改变底物结构, 同时也避免了预安装和除去导向 基团的步骤, 大大提高了反应的效率和相容性. 因此, 探索新颖的超分子催化方式无疑具有广阔的研究前景.

杯芳烃, 作为继冠醚和环糊精之后的第三代超分子 大环化合物, 具有环状的疏水性空腔, 能通过非共价键 与离子和中性分子形成稳定的包合物晶体结构, 从而使 其具有优异的选择性络合识别性能和相转移催化功 能 ${ }^{[11-12]}$. 目前, 杯芳烃及其衍生物已在离子载体 ${ }^{[13]}$ 、化 学分析 ${ }^{[14]}$ 、荧光传感 ${ }^{[15]}$ 、超分子自组装 ${ }^{[16]}$ 和分子识别 ${ }^{[17]}$ 等方面表现出广阔的应用前景. 在 Aldol 反应 ${ }^{[18]}$ 、 Michael 加成反应 ${ }^{[19]}$ 、 Henry 反应 ${ }^{[20]}$ 及选择性 Biginelli 反应 ${ }^{[21]}$ 等有机催化方面, 杯芳烃作为催化剂表现出了 优异的催化性能. 杯芳烃由于含有 $\pi$ 体系洞穴和多个紧 密相邻的酚氧原子, 杯芳烃与过渡金属离子[22]易于配 位, 与此同时在杯芳烃的上沿和下沿修饰中引入较软配
位原子的基团，可以进一步提高杯芳烃与过渡金属离子 的络合能力. 由于杯芳烃的特殊结构和过渡金属离子的 催化活性的结合，使杯芳烃过渡金属配合物具有结构稳 定、热稳定性好和选择性高等优势，使得杯芳烃过渡金 属配合物的发展越来越多样化. 目前, 杯芳烃化合物在 有机金属催化中的应用已呈现普遍上升趋势，且杯芳烃 过渡金属配合物已经被用于不同类型的过渡金属催化 反应，包括烯烃不对称氢化反应、胺化反应、环氧化反 应以及各类交叉偶联反应等. 本文将对最近十年来杯芳 烃参与的不同类型的过渡金属催化反应的研究进展分 两类介绍，包括杯芳烃促进的贵重过渡金属催化反应和 廉价过渡金属催化反应.

\section{1 杯芳烃促进的贵重过渡金属催化反应}

\section{1 杯芳烃-Rh 催化体系}

近年来，过渡金属催化是实现烯烃不对称氢化反应 的重要催化转化方法之一, 而手性膦配体在此类催化体 系中是最为关键的因素, 因此对新型手性膦配体的进一 步开发备受关注 ${ }^{[23]}$. 目前含有杯 [4]芳烃的手性膦配体 已经极大地丰富了配位化学的内容, 并且已经在过渡金 属催化反应中被广泛应用, 但应用于不对称催化的例子 却不多 ${ }^{[24-25]} .2010$ 年, Sandoval 课题组 ${ }^{[26]}$ 在杯 [4]芳烃下 沿引入手性膦配体, 制备了一系列手性二亚磷酸酯杯 [4]芳烃衍生物 $\mathbf{L 1} \sim \mathbf{L} 7$ ，并将这些手性杯 [4]芳烃膦配体 与贵重过渡金属铑反应，制得相应的杯 [4]芳烃铑配合 物, 然后将这些配合物作为催化剂用于烯烃的不对称氢 化反应(Scheme 1). 该加氢催化反应可以实现甲基乙酰
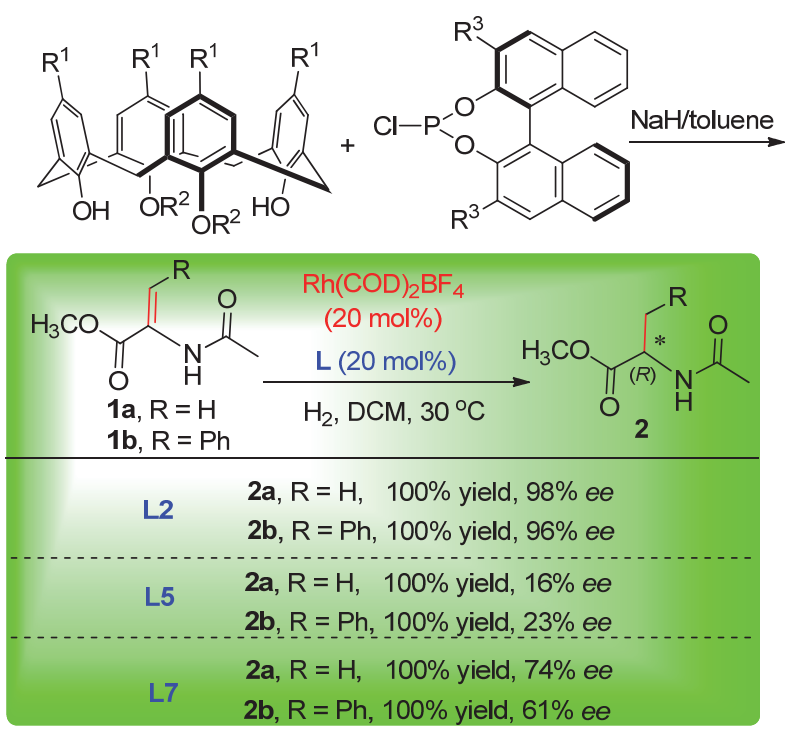

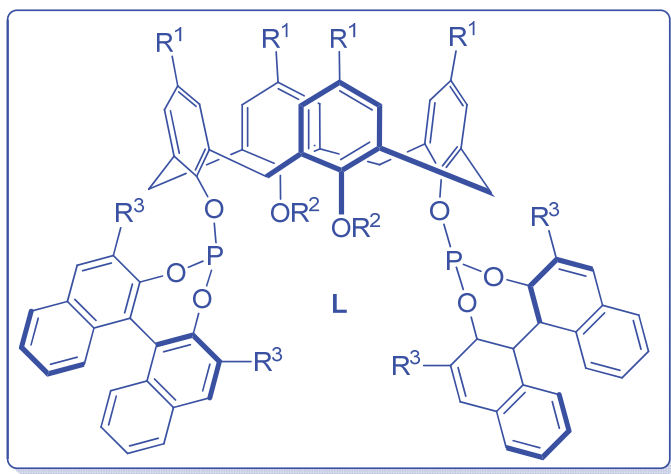

L1: $\mathrm{R}^{1}=\mathrm{C}\left(\mathrm{CH}_{3}\right)_{3}, \mathrm{R}^{2}=\mathrm{CH}_{3}, \mathrm{R}^{3}=\mathrm{H}$

L2: $\mathrm{R}^{1}=\mathrm{C}\left(\mathrm{CH}_{3}\right)_{3}, \mathrm{R}^{2}=\left(\mathrm{CH}_{2}\right)_{2} \mathrm{CH}_{3}, \mathrm{R}^{3}=\mathrm{H}$

L3: $\mathrm{R}^{1}=\mathrm{C}\left(\mathrm{CH}_{3}\right)_{3}, \mathrm{R}^{2}=\left(\mathrm{CH}_{2}\right)_{3} \mathrm{CH}_{3}, \mathrm{R}^{3}=\mathrm{H}$

L4: $\mathrm{R}^{1}=\mathrm{C}\left(\mathrm{CH}_{3}\right)_{3}, \mathrm{R}^{2}=\mathrm{CH}_{2} \mathrm{C}_{6} \mathrm{H}_{5}, \mathrm{R}^{3}=\mathrm{H}$

L5: $\mathrm{R}^{1}=\mathrm{C}\left(\mathrm{CH}_{3}\right)_{3}, \mathrm{R}^{2}=\mathrm{CH}_{2} \mathrm{C}_{6} \mathrm{H}_{5}, \mathrm{R}^{3}=\mathrm{CH}_{3}$

L6: $\mathrm{R}^{1}=\mathrm{H}, \mathrm{R}^{2}=\mathrm{CH}_{3}, \mathrm{R}^{3}=\mathrm{H}$

L7: $\mathrm{R}^{1}=\mathrm{CH}_{2} \mathrm{C}_{6} \mathrm{H}_{5}, \mathrm{R}^{2}=\mathrm{CH}_{2} \mathrm{C}_{6} \mathrm{H}_{5}, \mathrm{R}^{3}=\mathrm{H}$

图式 1 手性磷酸酯杯 [4]芳烃下铑催化不对称氢化反应

Scheme 1 Rhodium-catalyzed asymmetric hydrogenation in the presence of chiral phosphate calix[4]arene 
氨基丙烯酸酯以及与之相应的肉桂酸酯衍生物的还原. 实验结果表明，杯[4]芳烃上下沿连接基团的种类对反 应的催化活性有影响，当取代基 $\mathrm{R}^{1}$ 为叔丁基且 $\mathrm{R}^{2}$ 为正 丙基(L2)时, 产物 $\mathbf{2 a}$ 和 $\mathbf{2 b}$ 的 $e e$ 值分别为 $98 \%$ 和 $96 \%$.

2010 年, Matt 课题组 ${ }^{[27]}$ 利用利用配体 L2 和 L4 与 $\left[\mathrm{Rh}(\mathrm{acac})(\mathrm{CO})_{2}\right](\mathrm{acac}=$ 乙酰丙酮)反应制备了新颖的杯 芳烃催化剂 Cat-1 和 Cat-2, 并将其用于实现苯乙烯与 苄胺的氢胺化反应. 反应过程中可观察到该反应产生了 高比例的直链型亚胺, 其主要原因在于杯芳烃空腔和下 沿基团两者的结合使配体具有较宽的咬合角，同时空间 协同作用力使催化中心限制在紧密的分子袋中，这两个 特征都有利于形成具有线性结构的铑烷基中间体. 此 外, 无溶剂条件不会改变直链型产物的选择性, 同时还 会提高催化剂的活性, 有利于由伯胺选择性生成亚胺 (Scheme 2). 氢甲酰化反应是指在过渡金属催化剂的作 用下，在烯烃中通入一氧化碳和氢气合成醛的反应. 用 手性配体作催化剂实现的不对称氢甲酰化反应, 是一种 合成光学纯的支链醛的有效方法 ${ }^{[28]}$, 例如, 苯乙烯衍生 物通过不对称氢甲酰化反应，合成了生物活性分子布洛 芬和萘普生等 ${ }^{[29]}$. 最近, Sémeril 课题组 ${ }^{[30]}$ 用氢化钠对二 羟基杯 [4]芳烃进行双去质子化, 然后与 $(S)-(-)-1,1-$ 联 菜-2,2-二氧基氯磷反应合成二亚磷酸酯配体 L8. 该课 题组发现, $\left[\mathrm{Rh}(\mathrm{acac})(\mathrm{CO})_{2}\right]$ 与适量的配体 $\mathbf{L 8}$ 原位生成的 杯 [4]芳烃铑配合物可以实现苯乙烯及其衍生物的不对
称氢甲酰化反应(Scheme 3). 实验表明，在其它条件不 变的情况下, 降低底物苯乙烯反应量可以有效地提高反 应的转化率, 同时保持较高的 $e e$ 值. 值得注意的是, 在 苯乙烯的情况下, 支链醛为主产物是因为苯环的邻位碳 原子容易形成稳定的 $\eta^{3}$-烯丙基中间体.

2010 年, Harvey 课题组 ${ }^{[31]}$ 在杯[4]芳烃上沿引入手 性膦配体，合成了一种手性氨基膦-次膦酸酯杯 [4]芳烃 化合物 L9，具体合成方法如下：首先硼烷 3 与杯 [4]芳基 锂发生开环反应得到中间体 4 , 接着该中间体被氯二苯 膦捕获，与硼烷反应生成中间体 $\mathbf{5}$, 然后中间体 $\mathbf{5}$ 在保 留磷原子构型的基础上发生分解反应生成配体 L9. 该 课题组使用配体 $\mathbf{L 9}$ 与 $\left[\mathrm{Rh}(\mathrm{COD})_{2}\right] \mathrm{BF}_{4}(\mathrm{COD}=1,5$-环辛 二烯)反应合成配合物 Cat-3, 并将其作为催化剂用于烯 烃的不对称氢化反应. 杯芳烃上膦配体与铑催化剂络 合，导致金属催化中心偏向于空腔外部，这种构象极大 地增强了配合物 Cat-3 的不对称诱导性能. 实验结果表 明，配合物 Cat-3 具有优异的催化性能. 例如在 2-乙酰 氨基-3-苯基丙烯酸甲酯(6)的不对称氢化反应中，在 101 $\mathrm{kPa}$ 氢气压力和以苯为溶剂的情况下，配合物 Cat-3 可 以使原料高选择性地转化为产物 $(R)-N$-乙酰基-苯丙氨 酸甲酯(7), 其对映选择性为 $98 \% e e$. 此外，具有不同官 能团(酰胺基、甲基等)的烯烃底物也能以中等的 $e e$ 值得 到相应的不对称氢化产物(Scheme 4).

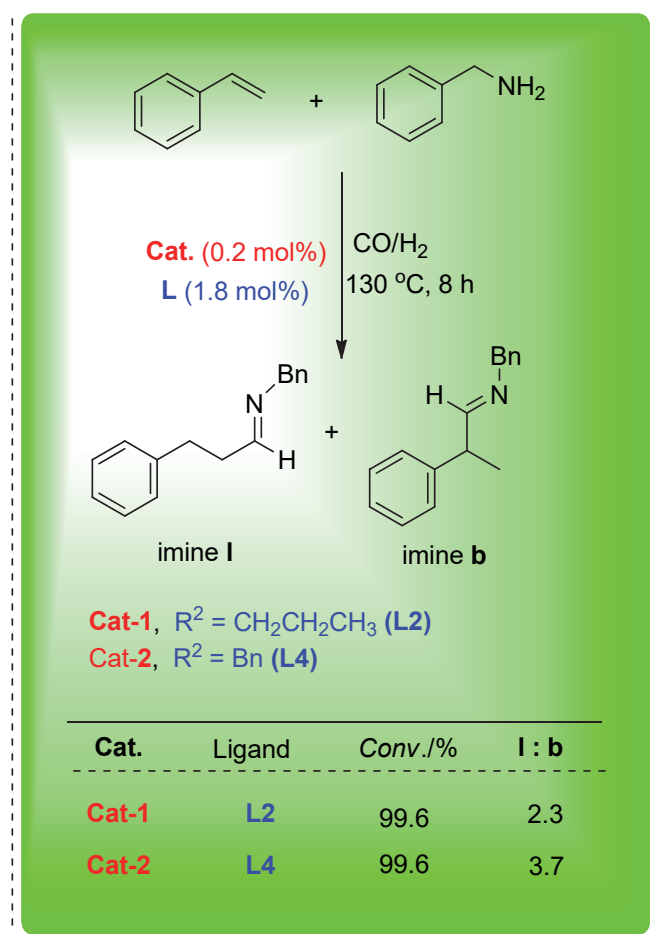

图式 2 磷酸酯杯[4]芳烃铑配合物催化氢胺化反应

Scheme 2 Phosphate calix[4]arene rhodium complex catalyzed hydrogen amination reaction 

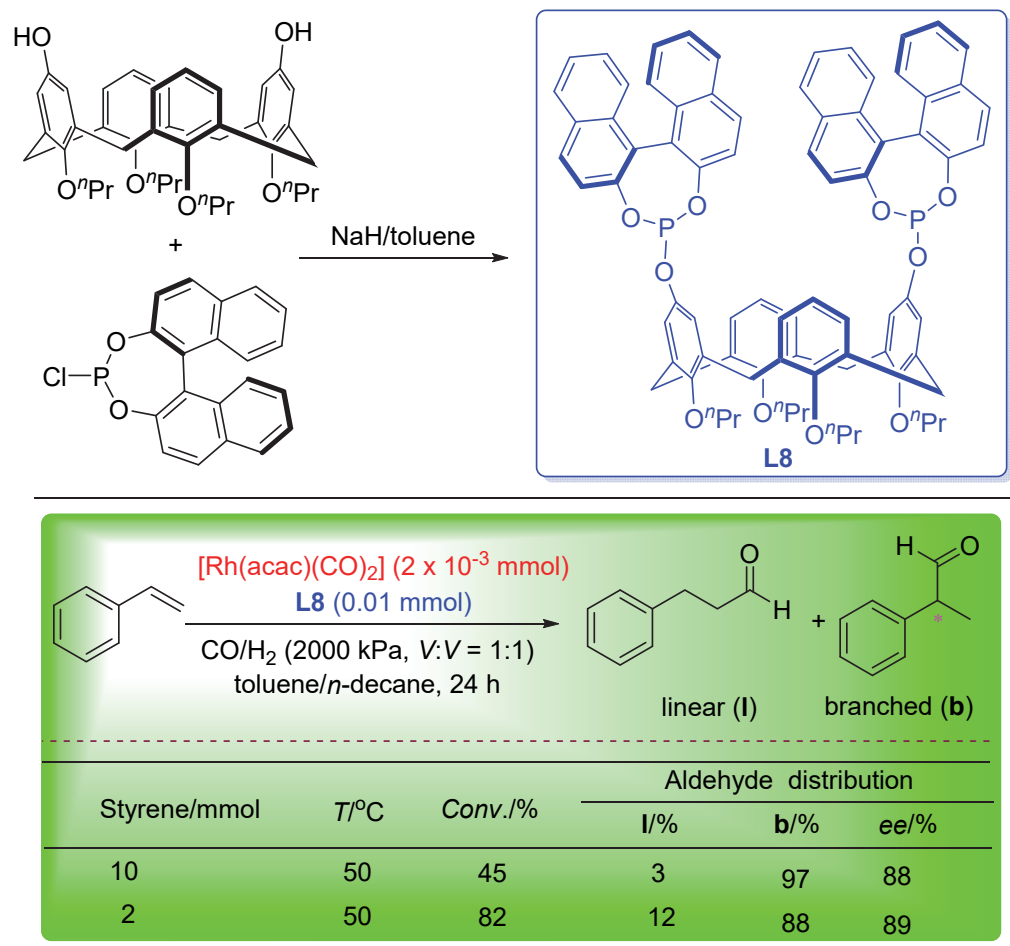

图式 3 二亚磷酸酯杯[4]芳烃下铑催化的乙烯基芳烃的不对称加氢甲酰化反应

Scheme 3 Asymmetric hydroformylation of vinylaromatics catalyzed by rhodium diphosphite calix[4]arene

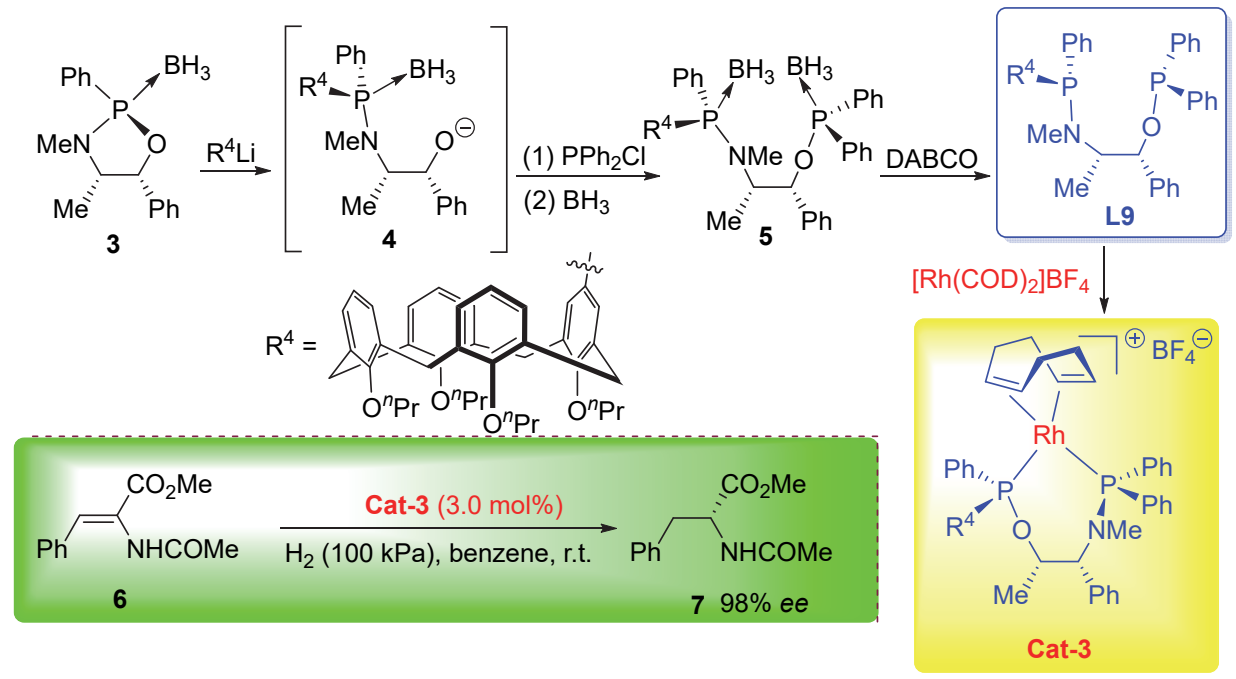

图式 4 手性氨基膦-次膦酸酯杯[4]芳烃铑配合物催化的烯烃不对称氢化反应

Scheme 4 Asymmetric hydrogenation of olefins catalyzed by chiral aminophosphine-phosphinate calix[4]arene rhodium complex

\section{2 杯芳烃-Pd 催化体系}

2013 年, Harvey 课题组 ${ }^{[32]}$ 同样在杯 [4]芳烃的上沿 引入磷原子制得单膦和双膦杯 [4]芳烃配体 L10 和 L11, 并将其作为过渡金属钯催化的配体来实现烯烃化合物 的 $\alpha$ 位的不对称取代反应. 实验结果表明, 双膦杯 [4]芳 烃配体 L11 催化效果优于单膦杯 [4]芳烃配体 L10. 以 $\left[\mathrm{Pd}\left(\mathrm{C}_{3} \mathrm{H}_{5}\right) \mathrm{Cl}\right]_{2}$ 为过渡金属催化剂, $\mathbf{L 1 1}$ 为配体, 可以实现 (E)-1,3-二苯基烯丙基乙酸酯 $(8)$ 和芐胺 9 的烯丙基胺化
反应，目标产物 $N$-芐基-1,3-二苯基-烯丙基胺(10)的对 映选择性为 $79 \% e e$ ，而在相同条件下使用配体 L10 时， 产物的对映选择性只有 $24 \%$ ee. 同时，在相同的催化体 系下，也可以实现 $(E)$-1,3-二苯基烯丙基乙酸酯 $(\mathbf{8})$ 与丙 二酸二甲酯(11)的不对称取代反应, 得到较高对映选择 性的目标产物 12 ( $82 \%$ ee) (Scheme 5).

Suzuki-Miyaura 偶联反应是通过过渡金属催化的芳 基卤化物和芳基硼酸构建不对称联芳基的反应，它已成 为构建众多复杂中间体的主要合成方法，包括天然产 

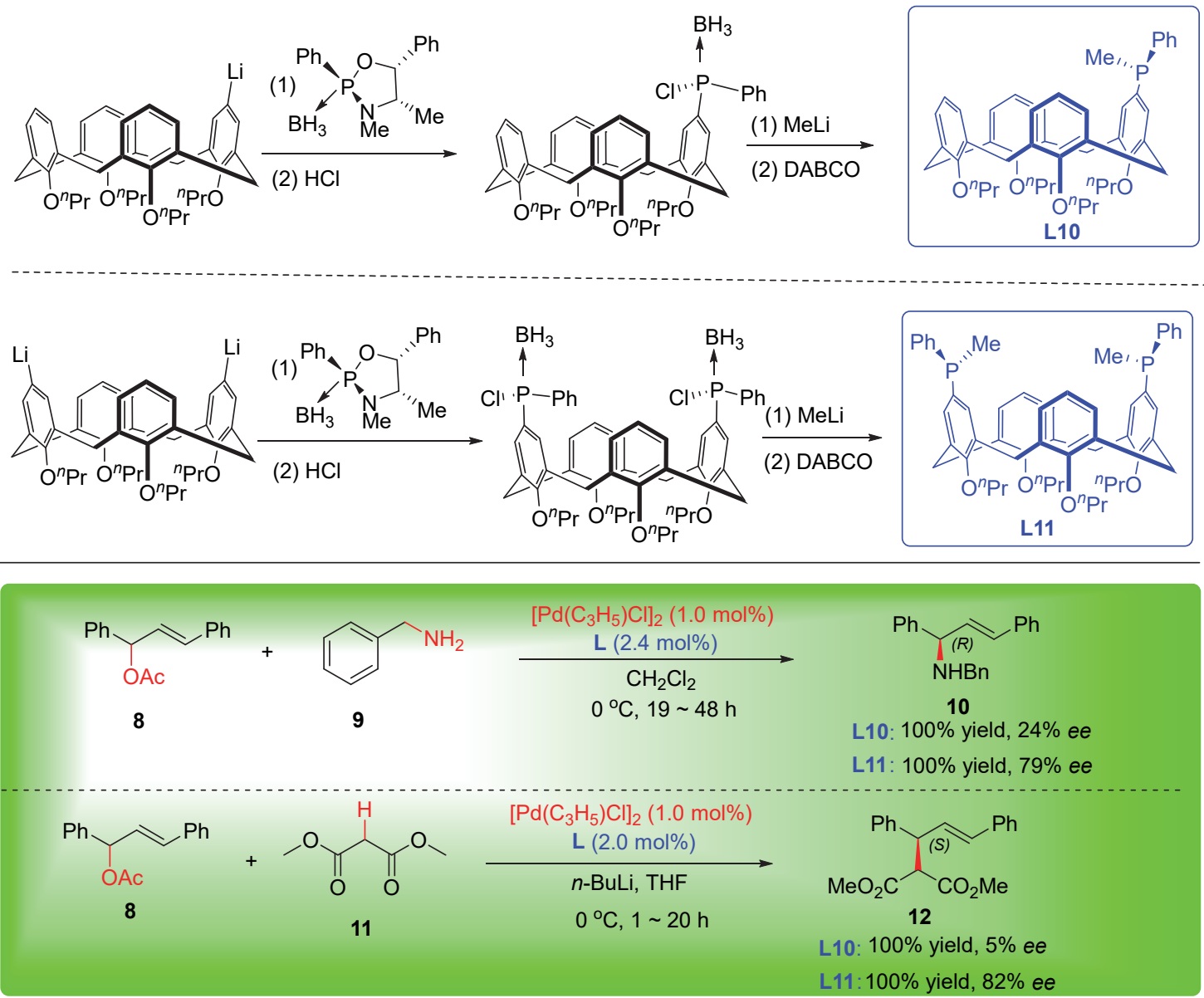

图式 5 杯 [4]芳烃配体 L10 和 L11 的合成及其与钯催化不对称的烯丙基胺化反应

Scheme 5 Synthesis of calix[4]arene ligands L10 and L11 and the asymmetric allyl amination catalyzed by palladium catalyst

物及生物活性分子 ${ }^{[33]} .2016$ 年, Hattori 课题组 ${ }^{[34]}$ 通过 Ullmann 型膦酰基化反应在 1,3-双(三氟甲磺酸酯)对叔 丁基杯 [4]芳烃的下沿引入双苯基膦基团，构建了杯 [4] 芳烃配体 $\mathbf{L 1 2}$, 然后 $\mathbf{L 1 2}$ 进一步与 $\mathrm{PdCl}_{2}(\mathrm{MeCN})_{2}$ 反应合 成了杯[4]芳烃钯催化剂 Cat-4 (Scheme 6). 此外, 该课 题组还研究了催化剂 Cat-4 在 Suzuki-Miyaura 偶联反应 以及环氧化物与苯硫酚的开环反应中的催化性能. 实验 结果表明, 该杯 [4]芳烃配合物对芳基氯化物和苯基硼 酸之间的 Suzuki-Miyaura 偶联反应显示出良好的催化活 性. 作者推测可能原因是, 在氢化钠的存在下, 苯硼酸 产生的苯基阴离子取代了两个乙腈的配体, 使 $\operatorname{Pd}(\mathrm{II})$ 更 容易转化为 $\operatorname{Pd}(0)$, 进而再通过还原消除得到目标产物. 与此同时, 在杯[4]芳烃化合物 Cat-4 催化 1,2-环氧辛烷 与苯硫酚的开环反应中, 只需要少量的催化剂就可以高 选择性地得到相应的 $\beta$-差基硫醚. 对于以上这些反应, 将三苯基膦 $\left(\mathrm{PPh}_{3}\right) 、( \pm)-2,2^{\prime}-$ 双-(二苯膦基)-1,1'-联菜 (BINAP)、1,3-双(二苯膦基)丙烷(dppp)和 1,3-双(二苯膦 基)丙烷( $\mathrm{dppf}$ )作为催化剂配体替代杯芳烃配体, 不能有 效地促进反应的进行. 此外, 该催化反应具有良好的官
能团相容性，但受一定的电子效应影响，除硝基外(硝 基与钯中心存在强配位，因此没有相应的偶合产物). 具有吸电子基团的芳基氯化物均能以良好的收率得到 相应的联芳基衍生物，而具有给电子基团(甲基)的芳基 氯化物在高温、高负载量催化剂作用下，其收率只有 $53 \%$. 值得注意, 在反应中加入 $\operatorname{Pd}(\mathrm{MeCN})_{4}\left(\mathrm{BF}_{4}\right)_{2}$ 和杯 芳烃配体 L12 代替催化剂 Cat-4, 并不能促进反应的进 行.

同年, Matt 课题组 ${ }^{[35]}$ 以 5-溴杯[4]芳烃为平台, 设计 并合成了 5-(2-二异丙基膦基苯基)-25,26,27,28-四丙基 氧 杯 [4] 芳 烃 (L13) 和 5-(2-二环已基膦基 苯 基)-25,26,27,28-四丙氧基杯 [4]芳烃(L14), 并将其用于 $\mathrm{Pd}_{2}(\mathrm{dba})_{3}(\mathrm{dba}=$ 二亚苄基丙酮)催化下芳基硼酸与芳基 氯化物的交叉偶联中反应. 实验结果表明, $\mathrm{Pd}_{2}(\mathrm{dba})_{3}$ 与 配体 L13 和 L14 原位生成的杯芳烃催化剂, 能够有效地 催化苯基硼酸与芳基氯化物的交叉偶联反应，同时该催 化反应受底物空间位阻影响较小, 均能以良好的收率实 现具有较大位阻的联芳基化合物的合成(Scheme 7). 


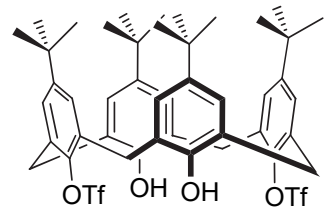

(1) Cul, $\mathrm{HP}(\mathrm{O}) \mathrm{Ph}_{2}$

(2) $\mathrm{HSiCl}_{3}, \mathrm{Et}_{3} \mathrm{~N}$

(3) $\mathrm{CH}_{3} \mathrm{l}, \mathrm{NaH}$

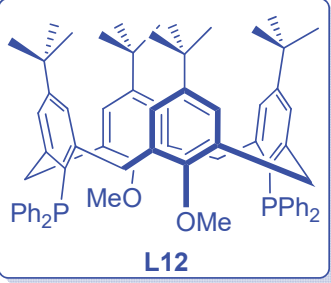

(1) $\mathrm{PdCl}_{2}(\mathrm{MeCN})_{2}$

(2) $\mathrm{AgBF}_{4}$

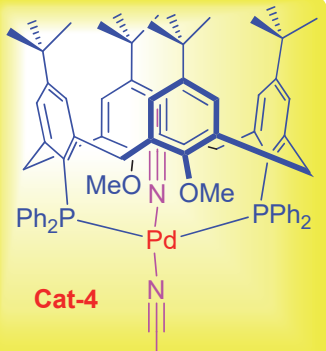

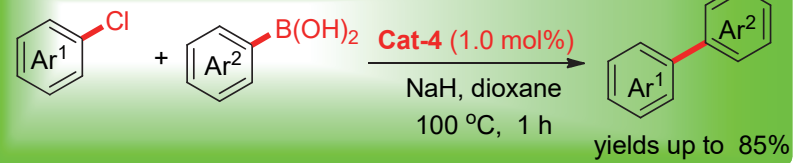

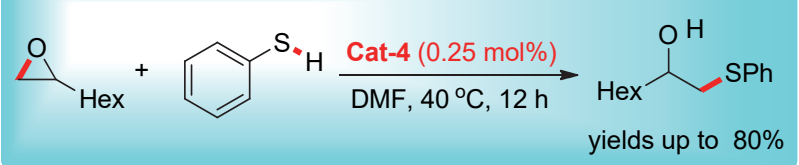

图式 6 杯[4]芳烃钯催化剂 Cat-4 催化的 Suzuki-Miyaura 偶联反应

Scheme 6 Suzuki-Miyaura coupling reaction catalyzed by calix[4]arene-palladium catalyst Cat-4

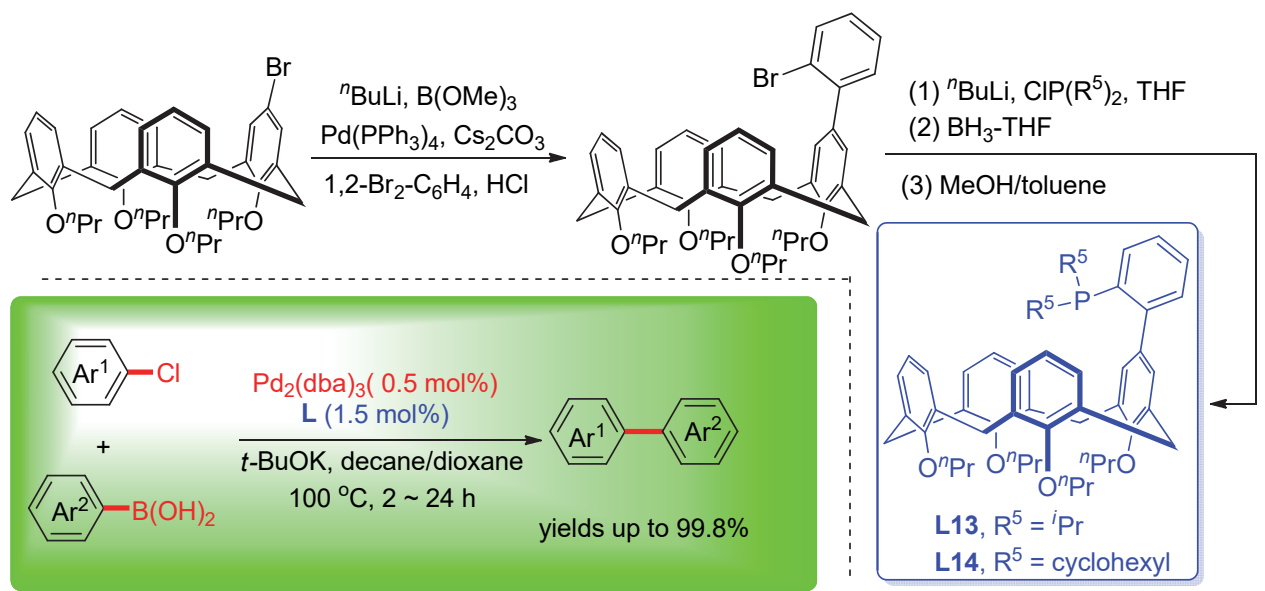

图式 7 杯[4]芳烃膦配体 L13 和 $\mathbf{L 1 4}$ 的制备及其在 Suzuki-Miyaura 偶联反应中的应用

Scheme 7 Preparation of calix[4]arene carbonyl ligands L13 and L14 and their application in Suzuki-Miyaura coupling reaction

目前，非均相过渡金属催化剂已经被广泛应用在碳 碳交叉偶联反应中 ${ }^{[36]} .2019$ 年, Rao 课题组 ${ }^{[37]}$ 基于介孔 二氧化硅(MCM-41)、四烯丙基杯[4]芳烃(allylCalix)和零 价钯纳米颗粒(Pd-NPs)合成了新型三元杂化物催化剂 Cat-5 (Pd@MCM-Calix ${ }_{\mathrm{ox}}$ ), 并利用该催化剂实现了碘苯 和苯基硼酸在水中的碳碳交叉偶联反应. 通过物理化学 技术表征发现，在没有使用任何外部还原剂的情况下， 四烯丙基杯[4]芳烃将 $\operatorname{Pd}(\mathrm{II})$ 还原成 $\operatorname{Pd}(0)$, 自身被氧化, 随后被氧化的杯[4]芳烃衍生物负载 $\operatorname{Pd}(0) \mathrm{NP}$ 得到三元 杂化物 Pd@MCM-Calix ${ }_{\text {ox }}$ (Scheme 8). 实验结果表明, 缺电子以及中性的苯基嗍酸衍生物具有良好的转化率 和选择性, 而富电子的苯基硼酸衍生物则会产生部分自 身偶联的产物. 该催化剂的高活性归因于杯芳烃衍生物 的辅助, 使 $\operatorname{Pd}(0) \mathrm{NPs}$ 可以良好分散. 另外, 由于杯状衍 生物的疏水性促进了底物分子在水中的吸附与扩散. 除 此之外, 该新型三元杂化物还被用于亲水和疏水硝基芳 族化合物的还原. 在常见的有机污染物和炸药中都含有
少量的硝基芳族化合物，将其转化为某些增值产品具有 重大的意义 ${ }^{[38]}$.

\section{2 杯芳烃促进的廉价过渡金属催化反应}

在过渡金属催化剂的研究中，相对于其它贵重金 属, 铜作为廉价过渡金属, 具有反应毒性低、价格低廉 及含量丰富等优势 ${ }^{[39]}$, 同时在绿色有机催化反应中铜 催化的研究也是一个重要方面, 因此到目前为止, 关于 杯[4]芳烃铜配合物催化剂的研究和报道越来越多. 此 外, 社会的可持续发展战略要求开发更为经济、环保、 高效的催化剂, 因此锰、镍等具有来源丰富、价格便宜 等优点, 有潜力成为贵金属的替代品，并发挥其自身独 特的反应性.

\section{1 杯芳烃-Mn 催化体系}

烯烃环氧化反应是有机合成和工业过程中非常重 要的反应 ${ }^{[40-41]}$. 在过去的几十年，已知廉价金属锰与 

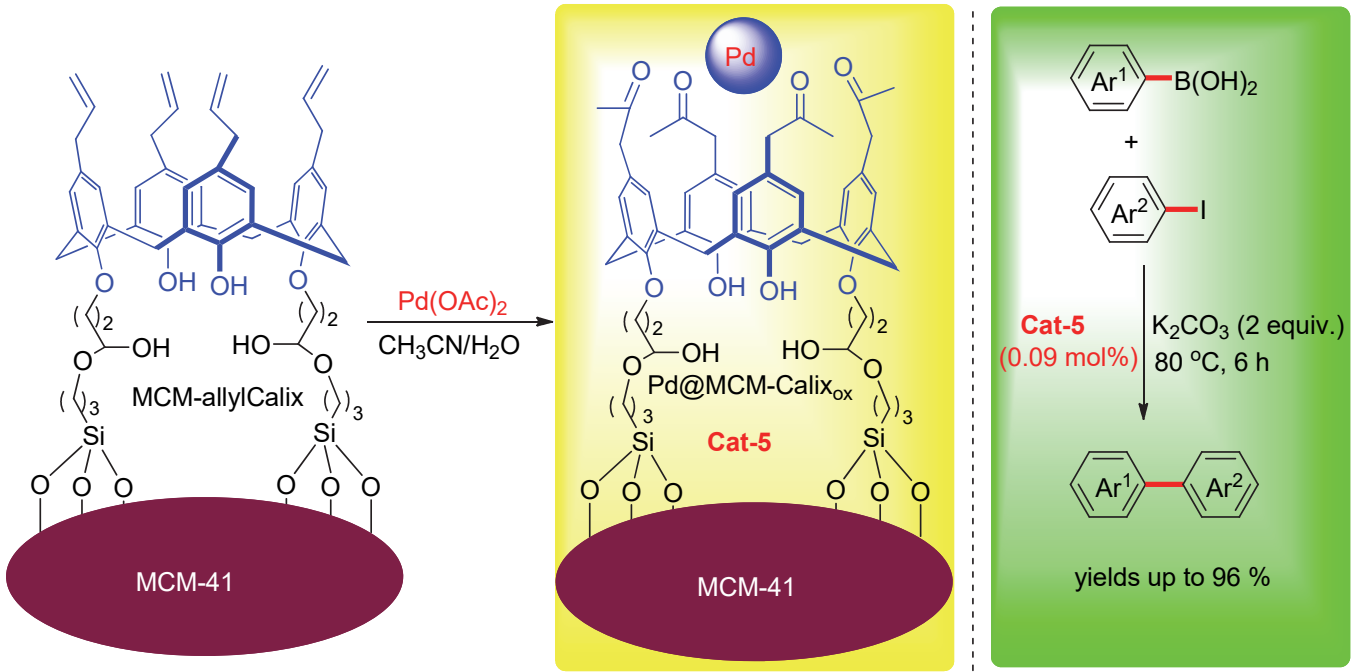

图式 8 三元杂化物杯[4]芳烃催化剂 Cat-5 催化的 C-C 交叉偶联反应

Scheme $8 \mathrm{C}-\mathrm{C}$ cross-coupling reaction catalyzed by ternary hybrid calix[4]arene catalyst Cat-5

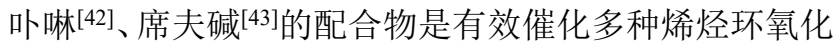
的催化剂, 但是这些催化剂存在选择性差及反应条件苛 刻等问题. 2011 年, 郎建平课题组 ${ }^{[44]}$ 以 5,11,17,23-四氨 基-25,26,27,28-四羟基杯 [4]芳烃为原料，通过与碘甲烷 反应构建了四羟基杯[4]芳烃四(三甲基铵)碘盐 L15, 然 后 $\mathbf{L 1 5}$ 再与 $\mathrm{Ag}(\mathrm{MeCN})_{4}\left(\mathrm{PF}_{6}\right)$ 反应得到相应的六氟磷酸 盐 L16. 由于 $\mathbf{L 1 6}$ 带有四个强吸电子的 $\mathrm{NMe}_{3}$ 正离子基 团, 增强了下沿酚羟基的酸性，可与四水合醋酸锰反应 制得杯 [4]芳烃催化剂 Cat-6. 结构上线性的锰过氧负离 子单元包含在杯芳烃空腔中, 其中杯芳烃空腔与氧原子 间的 $\pi \cdots \pi$ 相互作用、锰过氧负离子单元末端氧原子与
杯芳烃上沿 $\mathrm{NMe}_{3}$ 正离子基团的静电作用，以及与水分 子间的氢键作用，促进了超氧化物 Cat-6 的稳定性. 该 催化剂能够在温和条件下用氧气和异丁醛对环己烯进 行烯烃环氧化反应. 实验发现单独加入化合物 L16、四 水合醋酸锰或者不加任何催化剂时，产物的收率和立体 选择性都会大幅度降低. 此外, 实验结果还表明带有强 吸电子的 $\mathrm{NMe}_{3}$ 正离子基团可以提高环氧化反应的效 率，这可能是因为催化剂 Cat-6 中的 Mn(III)中心配位的 $\mathrm{H}_{2} \mathrm{O}$ 和过氧负离子易于离开，以及四个强吸电子 $\mathrm{NMe}_{3}$ 正离子基团的存在，使 $\mathrm{Mn}(\mathrm{III})$ 提供了用于环己烯的催 化环氧化的有效位点(Scheme 9).

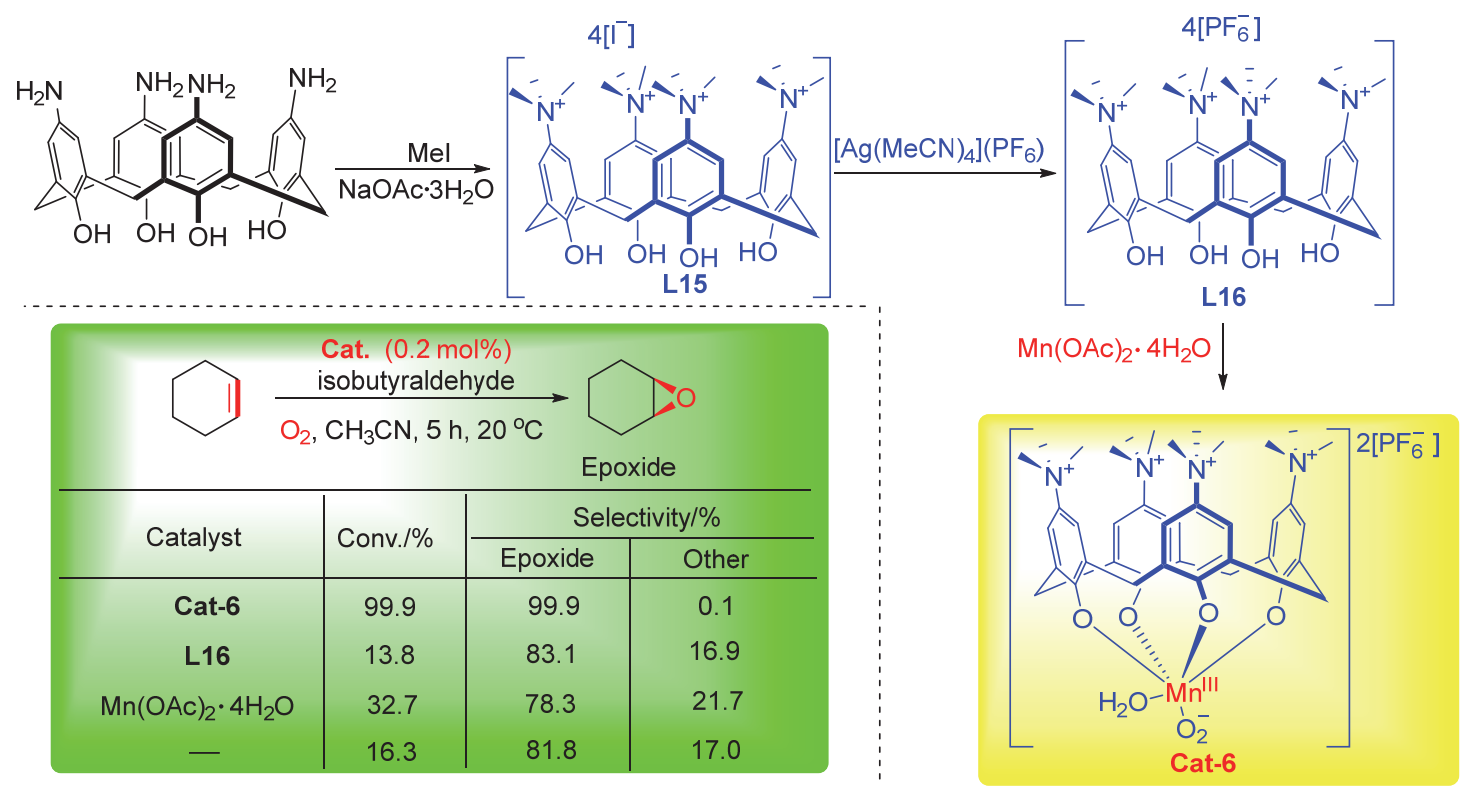

图式 9 含三甲基铵正离子杯 [4]芳烃锰催化剂 Cat-6 的制备以及在环己烯氧化反应中的应用

Scheme 9 Synthesis of calix[4]arene manganese catalyst containing trimethylammonium cation Cat-6 and its application in cyclohexene oxidation 
2014 年, Sciotto 课题组 ${ }^{[45]}$ 合成了基于 1,3-交替构象 的杯 [4]芳烃平台的两种杯 [4]芳烃-Mn 配合物, 并将其 作为烯烃双键的对映选择性环氧化反应的催化剂. 以 1,2-二澳乙烷或 1,3-二溴丙烷与 5,11-二丙氧基杯 [4]芳烃 为原料, 在碱的作用下获得相应的杯 [4]芳烃醚化产物, 然后与 2-烯丙氧基-3-羊基苯甲醛反应得到相应的中间 产物 13. 随后, 中间产物 13 脱去烯丙基保护基, 醛基与 手性 1,2-二苯基乙二胺反应得到相应的杯 [4]芳烃席夫 碱配体 L17 L18. 最后, 配体 $\mathbf{L 1 7}$ 和 $\mathbf{L 1 8}$ 与 $\mathrm{Mn}(\mathrm{OAc})_{3} \bullet$ $2 \mathrm{H}_{2} \mathrm{O}$ 反应制得催化剂 Cat-7 和 Cat-8. 以 6-甲氧基-2,2二甲基-1,2-二氢萗为底物进行不对称环氧化反应为例, 锰催化剂 Cat-7 显示出了满意的催化性能和立体诱导能 力, 反应的 $e e$ 值达到 $93 \%$, 而在相同的反应条件下使用 催化剂 Cat-7 时, 反应的收率几乎不变, 但是立体选择 性有所下降, ee 值只有 $66 \%$. 通过对催化剂结构的研究, 该课题组发现其二亚胺桥的两个立体中心靠近金属中 心，同时杯 [4]芳烃平台的存在提供了结合/催化位点, 这些条件都增强了该催化剂的对映选择性. 此外, 相同 条件下催化剂 Cat-7 比 Cat-8 催化效果更好, 可能是由 于杯芳烃上沿连接水杨酸的亚甲基的个数增多, 增加了 杯芳烃骨架与金属中心的距离, 从而不利于反应的立体 选择性控制(Scheme 10). 从反应结果上还可以发现, 环 状烯烃比非环状烯烃能更有效地进行对映选择性环氧 化反应，同时当底物具有吸电子基团时，其反应所产生 的环氧乙烷物质能在早期过渡态时与烯烃相互作用, 从 而提供较低的对映选择性值, 而具有给电子基团的则提 供较高的对映选择性值.

\section{2 杯芳烃-Cu 催化体系}

在过去的几十年中，聚 2,6-二甲基-1,4-亚苯基氧化 物(PPO)由于其优异的耐热性、电绝缘性及尺寸稳定性 等特点而备受关注 ${ }^{[46]}$. 由过渡金属 ${ }^{[47]}$ 或者酶催化 ${ }^{[48]}$ 的 苯酚氧化聚合反应是合成酚醛聚合物最有效的方法之 一. 在过渡金属配合物中, $\mathrm{Cu}(\mathrm{II})$ 配位化合物经常用于催 化 2,6-二甲基苯酚(DMP)氧化聚合, 但是因为这些聚合 反应通常在有机溶剂如甲苯 ${ }^{[49]}$ 和苯 ${ }^{[50]}$ 中进行, 溶剂需 要进行工艺回收, 这可能造成环境的污染并且增加生产 的成本，因此这种方法具有一些潜在的局限性. 2012 年, 郎建平课题组 ${ }^{[51]}$ 以 5,11,17,23-四 (三甲基铵)-25,26,27, 28-四羟基杯 [4]芳烃与水合醋酸铜反应，制备了水溶性 单核铜(II)催化剂杯[4]芳烃-Cu(II)配合物(Cat-9), 并研 究其在 2,6-二甲基苯酚氧化聚合反应中的催化性能 (Scheme 11). 杯芳烃上沿修饰的 NMe 正离子不仅增加 了所得配位化合物在水中的溶解度和稳定性, 而且还可 以作为水/有机双相体系中的相转移功能诱导基团. 实 验结果表明, 以 $\mathrm{O}_{2}$ 作氧化剂, 配合物在 2,6-二甲基苯酚 的氧化聚合中显示出高催化活性, 并以高收率获得了产 物聚 2,6-二甲基-1,4-亚苯基氧化物，同时几乎没有副产 物联苯醌的产生. 该课题组推测可能存在的反应机理 为: 首先 2,6-二甲基苯酚阴离子取代水分子并与杯 [4]芳 烃配合物的 $\mathrm{Cu}(\mathrm{II})$ 结合生成中间体 14. 随后中间体 $\mathbf{1 4}$ 可以经历分子内单电子转移产生中间体 $\mathbf{1 5}$. 而中间体 15 可以进行苯氧基和一个苯酚之间的偶联反应得到二 聚苯氧基的中间体 16. 与此同时, 聚合物链由此通过逐 步生长而增长, 而在聚合反应结束时, 聚合物链终止

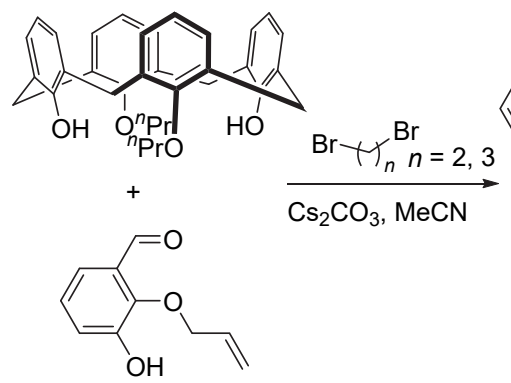

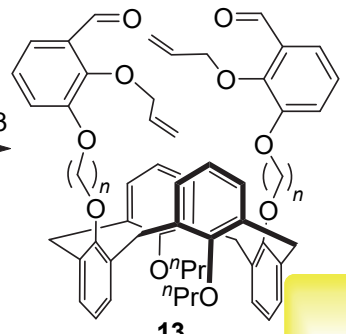

(1) $\mathrm{Pd}(\mathrm{OAc})_{2}, \mathrm{PPh}_{3}$ $\mathrm{Et}_{3} \mathrm{~N}, \mathrm{HOAC}$

(2) $P$

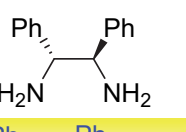

13
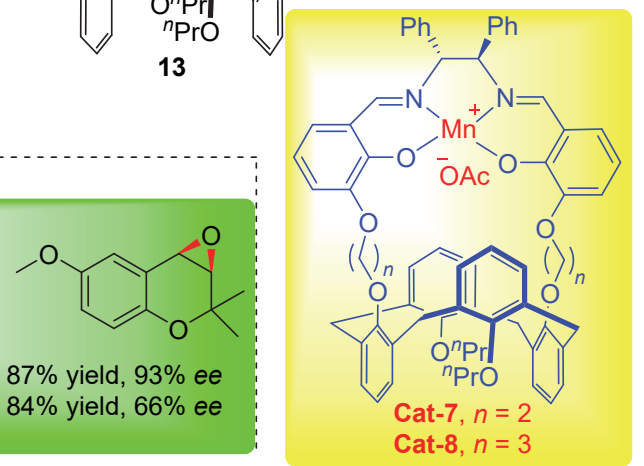

Cat-8, $n=3$

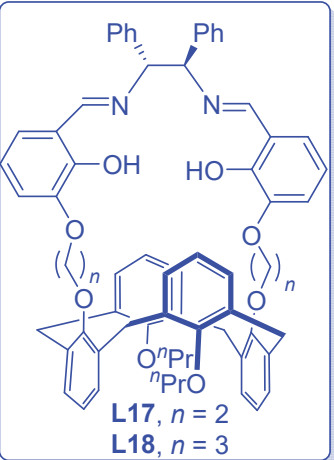

$\mathrm{Mn}(\mathrm{OAc})_{3} \cdot 2 \mathrm{H}_{2} \mathrm{O}$

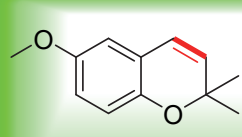

\begin{tabular}{l}
$\begin{array}{l}\text { Cat-7/Cat-8 } \\
\mathrm{NaClO}\end{array}$ \\
\hline $\mathrm{DCM} / \mathrm{H}_{2} \mathrm{O}, 0^{\circ} \mathrm{C}$
\end{tabular}

Cat-7, $87 \%$ yield, $93 \%$ e

Cat- $-8,84 \%$ yield, $66 \%$ ee

图式 10 1,3-交替构象的杯 [4]芳烃锰催化剂的合成以及在 6-甲氧基-2,2-二甲基-1,2-二氢萗环氧化反应中的应用

Scheme 10 Synthesis of calix[4]arene manganese catalyst in 1,3-alternating conformation and its application in epoxidation of 6methoxy-2,2-dimethyl-1,2-dihydronaphthalene 


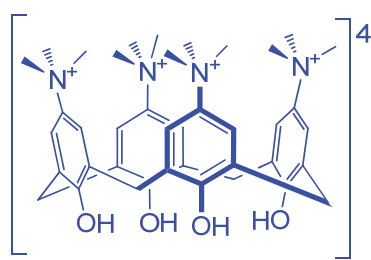

L15

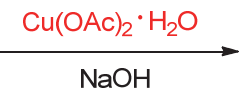

$\mathrm{NaOH}$
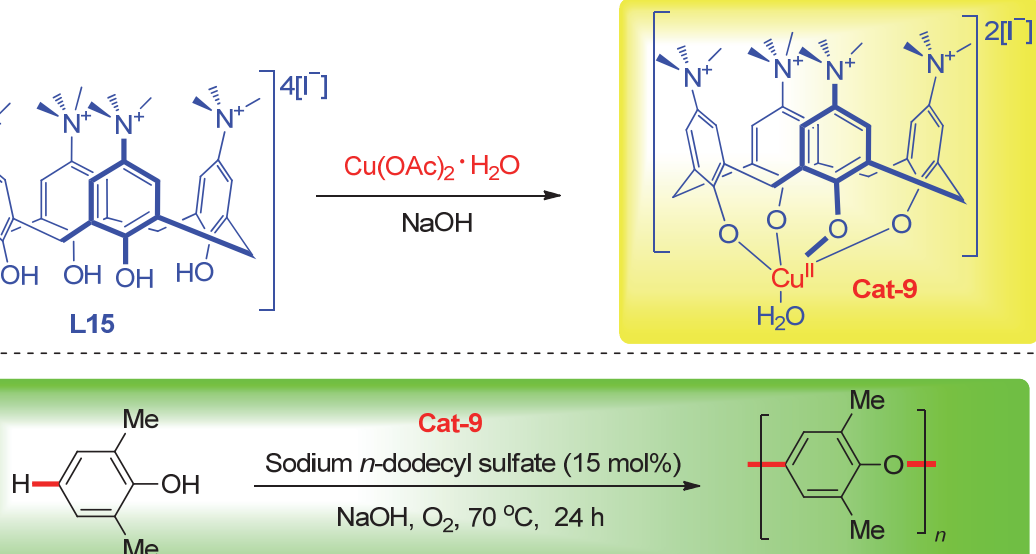

PPO $94 \%$ yield

图式 11 三甲基铵正离子杯 [4]芳烃铜催化剂 Cat-9 的合成以及在 2,6-二甲基苯酚氧化聚合反应中的应用

Scheme 11 Synthesis of trimethylammonium cation calix[4]arene copper catalyst Cat-9 and its application in the oxidation polymerization of 2,6-dimethylphenol

得到最终的聚 2,6-二甲基-1,4-亚苯基氧化物. 在聚合反 应结束时, $\mathrm{Cu}(\mathrm{I})$ 被过氧负离子氧化为 $\mathrm{Cu}(\mathrm{II})$ 中间体 $\mathbf{1 7}$, 而中间体 17 与 2,6-二甲基苯酚阴离子的反应使 $\mathrm{Cu}(\mathrm{I})$ 中 间体 14 再生，从而形成了反应的催化循环(Scheme 12).

杯芳烃的过渡金属配合物在各种水相反应中表现 出优异的催化性能 ${ }^{[52]}$, 但是在醇氧化反应中却很少被 报道. 2015 年, 郎建平小组 ${ }^{[53]}$ 将水溶性杯[4]芳烃 $\mathrm{Cu}(\mathrm{II})$
催化剂 Cat-9 用于苄醇的氧化反应，并且以良好的分离 收率获得了相应的醛类衍生物. 该种方法开辟了水溶性 铜配合物催化醇醛氧化的新途径. 优化反应条件发现, 反应体系催化剂 Cat-9/TEMPO $/ \mathrm{H}_{2} \mathrm{O} / \mathrm{K}_{2} \mathrm{CO}_{3}$ [TEMPO= 2,2,6,6-四甲基哌啶氮氧化物]非常适合于含有取代基的 茮醇，包括含杂原子的芳香醇、1-菜甲醇和 1,4-苯二甲 醇等的氧化. 但是该反应底物存在着空间位阻效应，邻

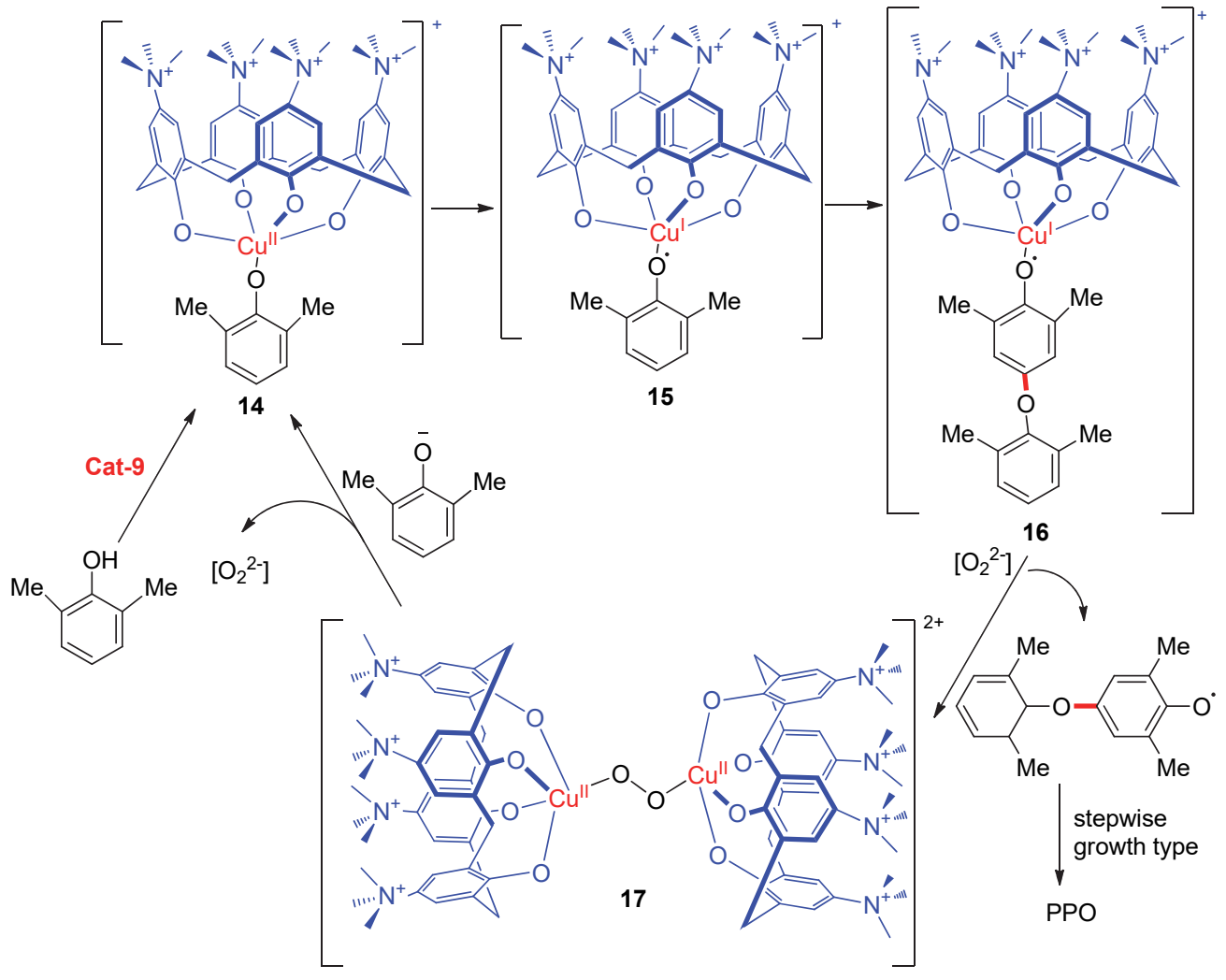

图式 12 2,6-二甲基苯酚氧化聚合反应的可能机理

Scheme 12 Proposed mechanism of oxidative polymerization of 2,6-dimethylphenol 
位取代基团的苄醇化合物所得相应目标产物的产率最 低. 此外, 该催化体系与其它 $\mathrm{Cu}(\mathrm{II})$ 催化剂/TEMPO 体 系相比，在同样的反应时间内能够得到更高的收率. 该 课题组提出了可能的反应机理: 首先, 在碳酸钾的作用 下, 芐醇脱去氢质子并与催化剂结合得到 $\mathrm{Cu}(\mathrm{II})$ 复合物 18. 之后, TEMPO 自由基作用于 $\mathrm{Cu}$ (II)复合物 18, 并在 氧负离子的邻位碳上夺得一个氢自由基生成茮基中间 产物 19. 在这一过程中, 由于配体 $\mathbf{L 1 5}$ 与 $\mathrm{Cu}(\mathrm{II})$ 的络合 可以避免 TEMPO 在 $\mathrm{Cu}(\mathrm{II})$ 中心的直接配位, 因此认为 此步骤很慢, 并且决定了整个反应的速率. 接下来的反 应步骤中, 通过还原 $\mathrm{Cu}(\mathrm{II})$ 中心将苄基自由基氧化为苯 甲醛, 并得到中间产物 20 , 而中间产物 20 可通过 TEMPO 自由基还原而重新氧化, 并结合水分子生成
Cu(II)催化剂 Cat-9 (Scheme 13a).

喹喔啉是一类有机含氮杂环化合物, 由于其具有抗 癌、抗微生物及抗生素等生物活性, 已被广泛用于药物 化学, 此外, 喹喔啉衍生物还被用作染料、发光材料、 半导体和腐蚀抑制剂等 ${ }^{[54-58]}$. 2017 年, 郎建平课题组 ${ }^{[59]}$ 研究了催化剂 Cat-9 在水相中催化 $\alpha$-苯基苯乙酮与 1,2苯二胺生成喹喔啉的催化性能. 虽然该方法中产物的产 率略微低于如 $N, N$-二甲基甲酰胺(DMF)和二甲基亚砜 (DMSO) 等有机溶剂中的，但是该反应使用水作为反应 溶剂更为绿色环保. 该反应是分两步进行的, 首先 $\alpha$-苯 基苯乙酮氧化为 1,2-二羰基化合物, 然后 1,2-二羰基化 合物与 1,2-苯二胺环化生成喹喔啉. 其中取代基的电子 性质对于氧化过程和环化过程的影响各不相同. 氧化过
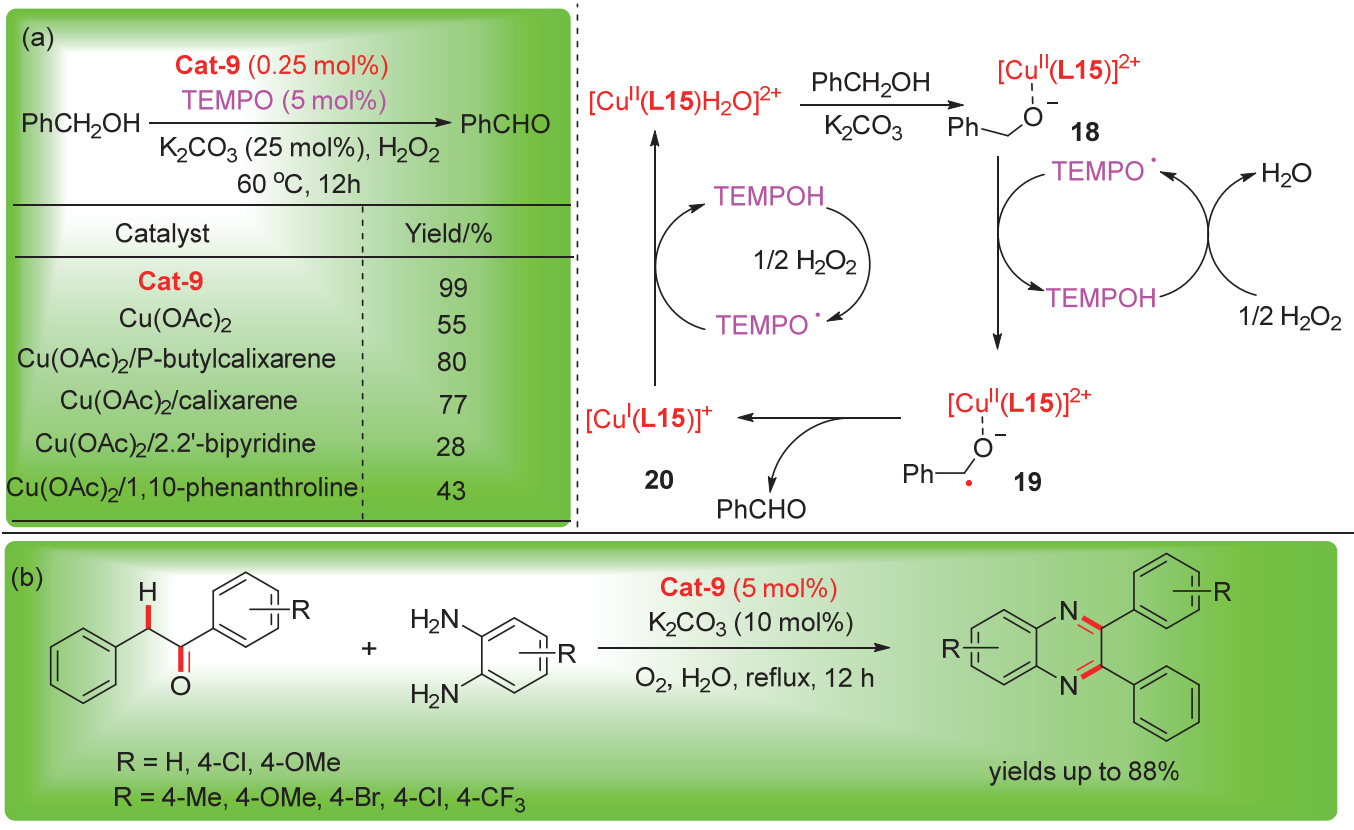

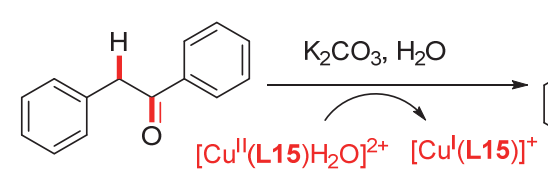

21

$\mathrm{O}_{2}$<smiles>O=C(Cc1ccccc1)c1ccccc1</smiles>

22<smiles>O=C(c1ccccc1)C(OO)c1ccccc1</smiles>

23

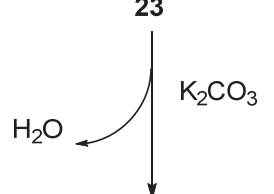<smiles>O=C(C(=O)c1ccccc1)c1ccccc1</smiles>

24

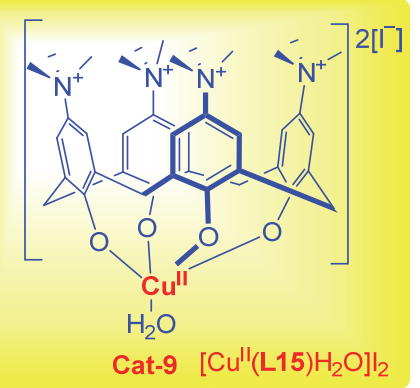<smiles>c1ccc(-c2nc3ccccc3nc2-c2ccccc2)cc1</smiles><smiles>Nc1ccccc1N</smiles><smiles>C1CCC1</smiles>

图式 13 三甲基铵正离子杯[4]芳烃铜催化剂 Cat-9 在苄醇的氧化反应以及喹喔啉制备中的应用

Scheme 13 Application of trimethylammonium cation calix[4] arene copper catalyst Cat-9 in the oxidation reaction of benzyl alcohol and the preparation of quinoxaline 
程中, 具有吸电子基团的苯基苯乙酮能以稍高的产率得 到相应氧化产物; 而在环化过程中则相反, 连有给电子 基团的 1,2-苯二胺的反应收率可达 $88 \%$, 优于具有吸电 子基团的 1,2-苯二胺(收率 76\% 78\%). 在最佳反应条 件下，体系 Cat-9/ $\mathrm{K}_{2} \mathrm{CO}_{3} / \mathrm{O}_{2} / \mathrm{H}_{2} \mathrm{O}$ 广泛适用于含有取代基 的 $\alpha$-苯基苯乙酮和芳香 1,2-二胺, 且最高收率可达 $88 \%$. 可能的反应机理如下: 在催化剂 Cat-9 的存在下, 先通 过氧化和去质子化将底物 $\alpha$-苯基苯乙酮转化为茮基自 由基 22, 该苠基自由基可被氧捕获以产生过氧化物基, 并捕获氢自由基产生中间产物 23 , 消除一分子的水后 生成 1,2-二羰基化合物 24, 中间产物 24 与 1,2-苯二胺缩 合并脱水得到相应的喹喔啉 25 (Scheme 13b).

除了以上报道的杯 [4]芳烃催化体系外, 杯[8]芳烃 衍生物也可用作过渡金属催化反应的配体. 2016 年 Castillo 课题组 ${ }^{[60]}$ 报道了第一例 $\mathrm{Cu}(\mathrm{I})$ 与含有菲咯啉的杯 [8]芳烃配体原位生成催化剂催化 $\mathrm{C}-\mathrm{S}$ 键偶联的反应 (Scheme 14). 他们在 1,5-酚位的杯 [8]芳烃上附加一个内 源性的菲罗啉基作为配体 $\mathbf{L 1 9}$, 然后与 $\mathrm{CuCl}$ 原位反应 制成催化剂 Cat-10, 实验发现, 在反应时间和偶联产物 的产率方面, 使用预先制备的催化剂和原位生成的催化 剂获得的反应结果几乎相同. 该催化反应体系要求产物 能够容易从杯芳烃结构中解离出来, 因此进行一系列的 溶剂篎选后发现, 较小极性的甲苯是较为有效的反应溶 剂, 这可能是因为甲苯中的非极性环境有利于物质进入 杯芳烃的空腔并且从空腔中释放出来. 其次, 该反应的 良好催化性能可直接归因于杯 [8]芳烃疏水腔内活性位 点的限制，与较小的杯 [4]或杯 [6]芳烃同系物不同，杯
[8]芳烃似乎提供了大小合适的疏水空腔来包封客体, 在稳定了反应中间体的同时还防止了系统中二聚体的 形成以及活性中心的失活. 在该催化反应中，含有供电 子基(甲基或甲氧基)和吸电子基(氰基或硝基)的芳基卤 化物都具有良好的反应兼容性，收率可高达 $95 \%$. 此外, 杯芳烃的空腔影响了底物的选择性，受阻较少的溴代芳 烃比碘代芳烃的反应速度快. 这些结果显示相对于其它 的一价铜促进的 $\mathrm{C}-\mathrm{S}$ 键偶联反应，该杯芳烃催化剂体 系突破了其高极性溶剂的限制，有效地促进了芳基硫醚 的形成，这种超分子策略代表了杯 [8]芳烃衍生物在过 渡金属催化反应转化的一种新方法, 为超分子催化剂在 有限空间内进行催化反应的研究提供了新思路.

\section{3 杯芳烃-Ni 催化体系}

2017 年, Matt 课题组 ${ }^{[61]}$ 在杯[4]芳烃的上沿引入二 芳基膦，合成了 5-二苯基膦基-25,26,27,28-四苄氧基杯 [4]芳烃(L20)和 5-二苯基膦基-25,26,27,28-四-(对茴香 基)甲氧基杯[4]芳烃(L21), 并用于室温下 $\mathrm{Ni}(\mathrm{COD})_{2}$ $(\mathrm{COD}=1,5$-环辛二烯) 催化苯嗍酸与芳基氯化物的交叉 偶联中反应(Scheme 15). 实验结果表明, $\mathrm{Ni}(\mathrm{COD})_{2}$ 与杯 [4]芳烃 L20 和 L21 配体原位生成的杯芳烃催化剂能够 有效地催化苯基嗍酸与芳基氯化物的交叉偶联反应，并 且它们催化反应的活性明显高于常规三芳基膦配体，该 反应具有良好的官能团相容性，且没有明显的电子效 应，连有供电子基团(甲氧基或甲基)或吸电子基团(氰 基、硝基或醛基)的芳基氯化物，都适用于目前的催化体 系与苯基硼酸反应，且得到高收率的目标产物. 此外,

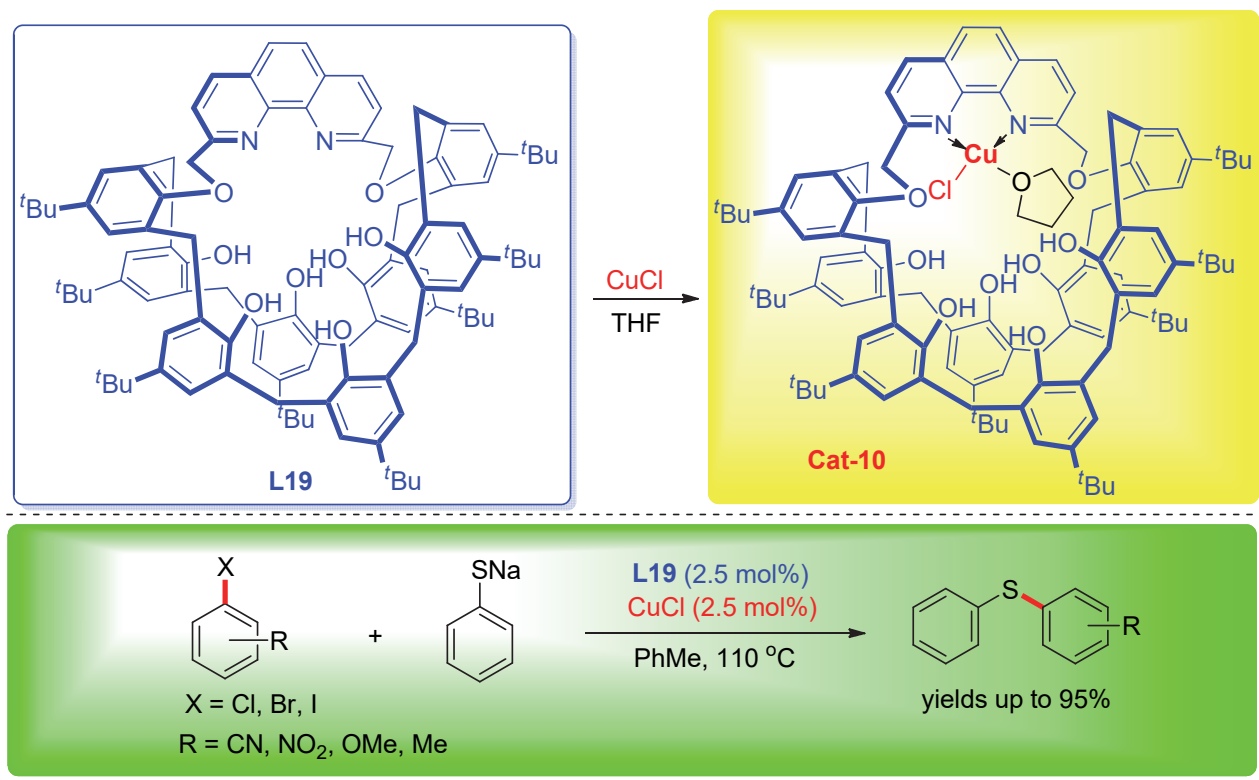

图式 14 菲咯啉杯 [8]芳烃配体 L19 促进下的铜催化 C-S 键偶联的反应

Sccheme 14 Copper-catalyzed $C-S$ bond coupling reaction promoted by phenanthroline calix[8] arene ligand L19 

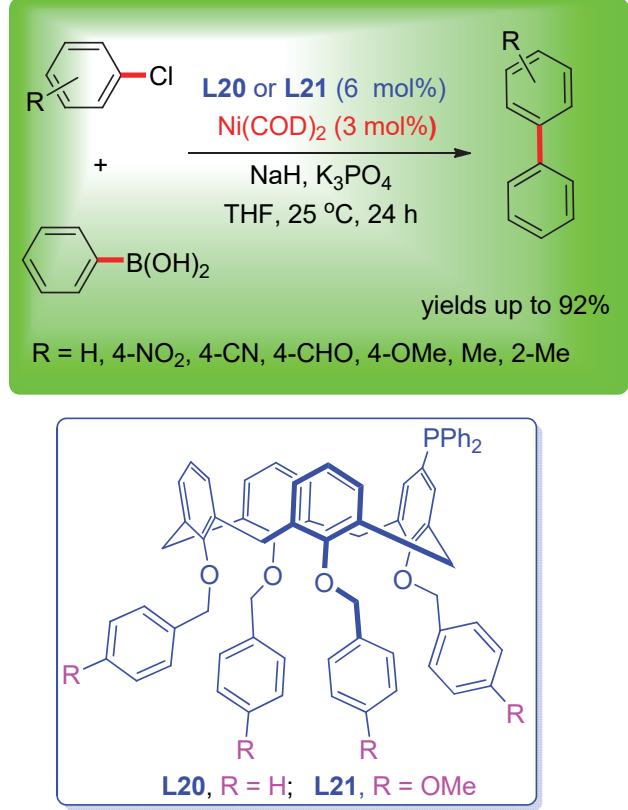

图式 15 二苯基膦基杯 [4]芳烃配体 L20 和 L21 促进的镍催化 交叉偶联反应

Scheme 15 Nickel-catalyzed cross-coupling reaction promoted by diphenylphosphino-calix[4]arene ligand $\mathbf{L 2 0}$ and $\mathbf{L 2 1}$

L20 和 L21 配体在该类反应体系中显示出了较好的热稳 定性和化学稳定性. 但是, 与经典的钯催化的交叉偶联 反应相比，镍催化的反应会产生大量的脱卤产物.

最近, Aloui 课题组 ${ }^{[62]}$ 将具有催化活性的 Ni-NHC 配 合物 $(\mathrm{NHC}=N$-杂环卡宾)与杯 $[6]$ 芳烃平台相连接, 合成 了多种新型过渡金属超分子催化剂 Cat-11、Cat-12 和
Cat-13. 该系列催化剂中 Ni-NHC 配合物是由 $1,3,5$-三甲 氧基-2,4,6-三取代的咪唑鎓三溴化物盐通过与镍茂反应 而合成，且 $\mathrm{NHC}$ 配体通过亚甲基间隔连接到 1,3,5-三甲 氧基对叔丁基杯 [6]芳烃的下边缘. 由于 $\mathrm{NHC}$ 配体是以 对称的形式连接到杯 [6]芳烃骨架上，因此催化剂提供 了理想的 $C 3$ 几何形状. 在探索该系列催化剂在交叉偶 联反应中的催化活性时，发现相对于芳基溴化物，芳基 氯化物反应较困难，但产率可达到 $90 \%$. 同时发现金属 中心与杯芳烃腔愈接近，即亚甲基链长愈短的情况下， 配合物的催化活性有所增强, 其中亚甲基链最长的催化 剂 Cat-13 催化收率仅有 84\%, 而亚甲基链相对较短的 催化剂 Cat-11 和 Cat-12 的催化收率则接近 100\% (Scheme 16).

\section{3 结论}

本文系统地综述了过去十年来杯芳烃参与的过渡 金属催化反应，主要参与的过渡金属有 $\mathrm{Rh} 、 \mathrm{Pd} 、 \mathrm{Mn}$ 、 $\mathrm{Cu}$ 、和 $\mathrm{Ni}$ ，涉及的反应有烯烃的不对称氢化、交叉偶联 反应、环氧化反应、聚合反应以及醇醛的氧化反应等. 在 这些工作中，一方面，通过对各种不同类型杯芳烃(包 括杯 [4]芳烃、杯 [6]芳烃和杯 [8]芳烃)下缘酚羟基、上缘 苯环对位以及连接苯环单元的亚甲基进行设计并改造, 可以有效地提高杯芳烃的超分子作用力以及其与过渡 金属的配位能力，从而促进过渡金属催化反应的进行; 另一方面，通过调控杯穴大小和功能基团的相对位置, 可以控制或者提升反应的立体选择性或区域选择性. 此 外，利用杯芳烃的疏水性能，引入亲水性功能基团，可
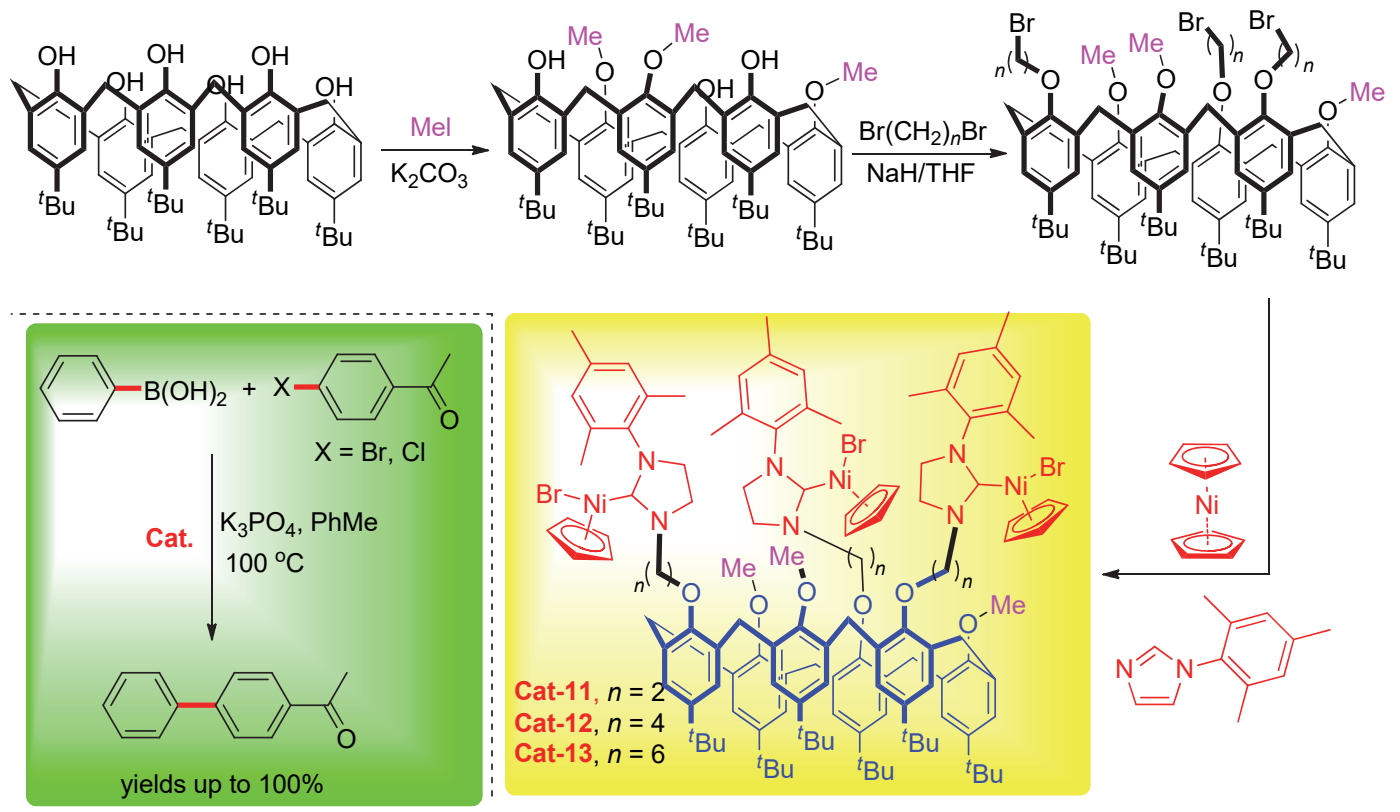

图式 16 杯[6]芳烃镍催化剂催化下的交叉偶联反应

Scheme 16 Cross-coupling reaction catalyzed by calix[6] arene nickel catalyst 
以拓展其相转移催化功能, 实现水相中的绿色催化反 应. 总之, 将杯芳烃超分子作用力与过渡金属的配位性 能有机结合, 可以更加有效地催化各类有机反应，从而 开发出系列多样化的基于杯芳烃平台的新型、高效、高 选择性的催化剂, 拓展杯芳烃应用领域的同时, 也为过 渡金属催化反应研究提供了新的思路和发展方向.

\section{References}

[1] (a) Ouyang, K.; Hao, W.; Zhang, W. X.; Xi, Z. Chem. Rev. 2015, $115,12045$.

(b) Bag, D.; Mahajan, S.; Sawant, S. D. Adv. Synth. Catal. 2020, 362,3948 .

(c) Li, H.; Ren, X. W.; Zhao, W. T.; Tang, X. Y.; Wang, G. W. Chin. J. Org. Chem. 2017, 37, 2287 (in Chinese).

(李桦, 任相伟, 赵温涛, 唐向阳, 王光伟, 有机化学, 2017, 37, 2287.)

[2] (a) Ansari, R. M.; Bhat, B. R. J. Chem. Sci. 2017, 129, 1483.

(b) Li, R. Q.; Xu, H.; Zhao, N.; Jin, X. J.; Dang, Y. F. J. Org. Chem. 2020, 85,833

(c) Chen, G. J.; Du, J. S. Chin. J. Org. Chem. 2014, 34, 65 (in Chinese).

(陈国军, 杜建时, 有机化学, 2014, 34, 65.)

(d) Han, F. S. Chem. Soc. Rev. 2013, 42, 5270

[3] Zhang, Q.; Muhammad, M. T.; Chiou, M. F.; Jiao, Y.; Bao, H.; Li, Y. Org. Lett. 2020, 22, 5261 .

[4] (a) Dieleman, C.; Steyer, S.; Jeunesse, C.; Matt, D. J. Chem. Soc., Dalton Trans. 2001, 17, 2508.

(b) Ma, X. T.; Yu, J.; Wang, Z. L.; Zhang, Y.; Zhou, Q. J. Chin. J. Org. Chem. 2020, 40, 2669 (in Chinese).

(马献涛, 于静, 王子龙, 张望, 周秋菊, 有机化学, 2020, 40, 2669.)

(c) Deng, Y. Y.; Yang, W.; Yang, X.; Yang, D. Q. Chin. J. Org. Chem. 2017, 37, 3039 (in Chinese).

(邓颖颖，杨文，杨新，杨定乔，有机化学，2017，37, 3039.)

(d) Yang, K.; Song, M. J.; Ma, Z. Y.; Li, Y.; Li, Z. Y.; Sun, X. Q.

Org. Chem. Front. 2019, 6, 3996.

(e) Yang, K.; Niu, B.; Ma, Z. Y.; Wang, H.; Lawrence, B.; Ge, H. B. J. Org. Chem. 2019, 84, 14045.

[5] (a) Wang, D. H.; Engle, K. M.; Shi, B. F.; Yu, J. Q. Science 2010, $327,315$.

(b) Bras, J. L.; Muzart, J. Chem. Rev. 2011, 111, 1170.

(c) Chen, F.; Wang, T.; Jiao, N. Chem. Rev. 2014, 114, 8613.

[6] (a) Lyons, T. W.; Sanford, M. S. Chem. Rev. 2010, 110, 1147. (b) Arockiam, P. B.; Bruneau, C.; Dixneuf, P. H. Chem. Rev. 2012, 112,5879 .

(c) Liu, C.; Yuan, J.; Gao, M.; Tang, S.; Li, W.; Shi, R.; Lei, A. Chem. Rev. 2015, 115, 12138 .

[7] Yang, H.; Yuan, B.; Zhang, X.; Scherman, O. A. Acc. Chem. Res. 2014, 47, 2106.

[8] Delbianco, M.; Bharate, P.; Varela-Aramburu, S.; Seeberger, P. H. Chem. Rev. 2016, 116, 1693.

[9] Dydio, P.; Reek, J. N. H. Chem. Sci. 2014, 5, 2135

[10] (a) Niu, B.; Yang, K.; Lawrence, B.; Ge, H. B. ChemSusChem 2019, 12, 2955.

(b) Yang, K.; Song, M. J.; Liu, H.; Ge, H. B. Chem. Sci. 2020, 11, 12616.

[11] Gutsche, C. D.; Dhawan, B.; No, K. H.; Mutbukrishnan, R. J. Am. Chem. Soc. 1981, 103, 3782

[12] Salvio, R.; Volpi, S.; Cacciapaglia, R.; Sansone, F.; Mandolini, L.; Casnati, A. J. Org. Chem. 2016, 81, 9012.

[13] Song, S. F. D.; Shang, X. F.; Zhao, J. L.; Hu, X. J.; Koh, K.; Wang, K. M.; Chen, H. X. Sens. Actuators, B 2018, 267, 357.

[14] (a) Hrdlička, V.; Navrátil, T.; Barek, J.; Ludvík, J. J. Electroanal. Chem. 2018, 821, 60 . (b) Li, L.; Qi, W. G.; Wang, C.; Yan, C. G. Chin. J. Org. Chem. 2013, 33, 1804 (in Chinese).

(李亮，戚伟光，王超，颜朝国，有机化学，2013，33，1804.)

[15] (a) Li, Z. Y.; Su, H. K.; Tong, H. X.; Yin, Y.; Xiao, T.; Sun, X. Q.; Jiang, J. L.; Wang, L. Y. Spectrochim. Acta, Part A 2018, 200, 307. (b) Zhang, C. L.; Gong, S. L.; Chen, Y. Y. Chin. J. Org. Chem. 2007, 27, 795 (in Chinese).

(张春雷, 龚淑玲, 陈远荫, 有机化学, 2007, 27, 795.)

(c) Liu, L. L.; Zhang, Z. G.; Li, H. X.; Shang, H.; Lang, J. P. Chin. J. Chem. 2010, 28, 1829.

[16] (a) Wang, Y. X.; Zhang, Y. M.; Liu, Y. J. Am. Chem. Soc. 2015, 137, 4543.

(b) Zhao, B. T.; Zhang, H, Y.; Liu, Y. Chin. J. Org. Chem. 2005, 25, 913 (in Chinese).

(赵邦屯, 张衡益, 刘育, 有机化学, 2005, 25, 913.)

(c) Liu, L. L.; Ren, Z. G.; Zhu, L. W.; Wang, H. F.; Yan, W. Y.; Lang, J. P. Cryst. Growth Des. 2011, 11, 3479.

[17] (a) Li, Z. Y.; Zhou, K.; Lai, Y.; Sun, X. Q.; Wang, L. Chin. J. Org. Chem. 2015, 35, 1531 (in Chinese)

(李正义, 周坤, 来源, 孙小强, 王乐勇, 有机化学, 2015, 35, 1531.)

(b) Liu, L. L.; Ren, Z. G.; Wan, L. M.; Ding, H. Y.; Lang, J. P. CrystEngComm 2011, 13, 5718.

[18] Li, Z. Y.; Chen, Y.; Zheng, C. Q.; Yin, Y.; Wang, L. Y.; Sun, X. Q. Tetrahedron 2017, 73, 78.

[19] (a) Li, Z. Y.; Tong, H. X.; Chen, Y.; Su, H. K.; Xiao, T. X.; Sun, X. Q.; Wang, L. Y. Beilstein J. Org. Chem. 2018, 14, 1901.

(b) Durmaz, M.; Sirit, A. Supramol. Chem. 2013, 25, 292.

(c) Yang, K.; Ma, Z. Y.; Tong, H. X.; Sun, X. Q.; Hu, X. Y.; Li, Z. Y. Chin. Chem. Lett. 2020, 12, 3259.

[20] Chang, M. L.; He, Y.; Zhou, J.; Li, S. Y. Chem. Soc. 2017, 28, 1363.

[21] Li, Z. Y.; Xing, H. J.; Huang, G. L.; Sun, X. Q.; Jiang, J. L.; Wang, L. Y. Sci. China: Chem. 2011, 54, 1726.

[22] (a) Cacciapaglia, R.; Casnati, A.; Mandolini, L.; Reinhoudt, D. N.; Salvio, R.; Sartori, R.; Ungaro, R. J. Am. Chem. Soc. 2006, 128 , 12322.

(b) Cacciapaglia, R.; Casnati, A.; Mandolini, L.; Ungaro, R. J. Chem. Soc., Chem. Commun. 1992, 1291.

[23] Tang, W. J.; Zhang, X. M. Chem. Rev. 2003, 103, 3029.

[24] (a) Gramage-Doria, R.; Armspach, D.; Matt, D. Coord. Chem. Rev. 2013, 257, 776 .

(b) Homden, D. M.; Redshaw, C. Chem. Rev. 2008, 108, 5086

[25] (a) Lai, S. W.; Chan, Q. K. W.; Han, J.; Zhi, Y. G.; Zhu, N. Y.; Che, C. M. Organometallics 2009, 28, 34.

(b) Sameni, S.; Lejeune, M.; Jeunesse, C.; Matt, D.; Welter, R. Dalton Trans. 2009, 38, 7912.

[26] Liu, S. S.; Sandoval, C. A. J. Mol. Catal. A: Chem. 2010, 325, 65.

[27] Monnereau, L.; Sémeril, D.; Matt, D. Green Chem. 2010, 12, 1670.

[28] (a) Adint, T. T.; Wong, G. W.; Landis, C. R. J. Org. Chem. 2013, 78 , 4231.

(b) Xu, K.; Zheng, X.; Wang, Z. Y.; Zhang, X. M. Chemistry 2014, 20, 4357.

(c) Rovira, L.; Vaquero, M.; Vidal-Ferran, A. J. Org. Chem. 2015, 80, 10397.

[29] Chaudhari, R. V. Top. Catal. 2012, 55, 439.

[30] Natarajan, N.; Pierrevelcin, M. C.; Semeril, D.; Bauder, C.; Matt, D.; Ramesh, R. Catal. Commun. 2019, 118, 70.

[31] Khiri, N.; Bertrand, E.; Ondel-Eymin, M. J.; Rousselin, Y.; Bayardon, J.; Harvey, P. D.; Juge, S. Organometallics 2010, 29, 3622.

[32] Khiri-Meribout, N.; Bertrand, E.; Bayardon, J.; Eymin, M. J.; Rousselin, Y.; Cattey, H.; Fortin, D.; Harvey, P. D.; Juge, S. Organometallics 2013, 32, 2827.

[33] Miyaura, N.; Suzuki, A. Chem. Rev. 1995, 95, 2457.

[34] Hirasawa, K.; Tanaka, S.; Horiuchi, T.; Kobayashi, T.; Sato, T.; Morohashi, N.; Hattori, T. Organometallics 2016, 35, 420.

[35] Elaieb, F.; Hedhli, A.; Sémeril, D.; Matt, D. Eur. J. Org. Chem. 2016, 2016, 1867.

[36] Zhang, F.; Chen, M. Z.; Wu, X. T.; Wang, W.; Li, H. X. J. Mater. 
Chem. A 2014, 2, 484

[37] Narkhede, N.; Uttam, B.; Rao, C. P. ACS Omega 2019, 4, 4908.

[38] (a) Zhang, Y.; Zhu, P. L.; Chen, L.; Li, G.; Zhou, F. R.; Lu, D. Q.; Sun, R.; Zhou, F.; Wong, C. P. J. Mater. Chem. A 2014, 2, 11966. (b) Hu, X. W.; Long, Y.; Fan, M. G.; Yuan, M.; Zhao, H.; Ma, J. T.; Dong, Z. P. Appl. Catal., B 2019, 244, 25.

[39] (a) Ramakrishna, D.; Bhat, B. R. Inorg. Chem. Commun. 2011, 14, 690.

(b) Rezaeifard, A.; Jafarpour, M.; Naeimi, A.; Salimi, M. Inorg. Chem. Commun. 2012, 15, 230.

[40] Lane, B. S.; Burgess, K. Chem. Rev. 2003, 103, 2457.

[41] Nehru, K.; Kim, S. J.; Kim, I. Y.; Seo, M. S.; Kim, Y.; Kim, S. J.; Kim, J.; Nam, W. Chem. Commun. 2007, 4623.

[42] Leeladee, P.; Goldberg, D. P. Inorg. Chem. 2010, 49, 3083.

[43] Cavallo, L.; Jacobsen, H. J. Org. Chem. 2003, 68. 6202.

[44] Liu, L. L.; Li, H. X.; Wan, L. M.; Ren, Z. G.; Wang, H. F.; Lang, J. P. Chem. Commun. 2011, 47, 11146.

[45] Bonaccorso, C.; Brancatelli, G.; Ballistreri, F. P.; Geremia, S.; Pappalardo, A.; Tomaselli, G. A.; Toscano, R. M.; Sciotto, D. Dalton. Trans. 2014, 43, 2183

[46] Sadeghi, F.; Tremblay, A. Y.; Kruczek, B. J. Appl. Polym. Sci. 2008, 109, 1454.

[47] (a) Liu, Q.; Shentu, B.; Gu, C.; Weng, Z. Eur. Polym. J. 2009, 45, 1080 .

(b) Kobayashi, S.; Higashimura, H. Prog. Polym. Sci. 2003, 28, 1015 .

(c) Villabrille, P.; Romanelli, G.; Vázquez, P.; Cáceres, C. Appl. Catal., A 2008, 334, 374.

[48] Kobayashi, S.; Makino, A. Chem. Rev. 2009, 109, 5288.

[49] (a) Liu, Y. R.; Li, J. P.; Hou, H. W.; Fan, Y. T. J. Organomet. Chem. 2009, 694, 2875. (b) Liao, B. S.; Liu, Y. H.; Peng, S. M.; Liu, S. T. Dalton Trans. 2012, 41, 1158 .

[50] (a) Bae, B.; Kawamura, S.; Miyatake, K.; Watanabe, M. J. Polym. Sci., Part A: Polym. Chem. 2011, 49, 3863.

(b) Liu, Q.; Shentu, B. Q.; Zhu, J. H.; Weng, Z. X. J. Appl. Polym. Sci. 2007, 104, 3649.

[51] Wan, L. M.; Li, H. X.; Zhao, W.; Ding, H. Y.; Fang, Y. Y.; Ni, P. H.; Lang, J. P. J. Polym. Sci., Part A: Polym. Chem. 2012, 50, 4864.

[52] Patel, R. V.; Panchal, J. G.; Menon, S. K. J. Inclusion Phenom. Macrocyclic Chem. 2010, 67, 63.

[53] Gao, J.; Ren, Z. G.; Lang, J. P. J. Organomet. Chem. 2015, 792, 88.

[54] Baudy, R. B.; Greenblatt, L. P.; Jirkovsky, I. L.; Conklin, M.; Russo, R. J.; Bramlett, D. R.; Emrey, T. A.; Simmonds, J. T.; Kowal, D. M.; Stein, R. P.; Tasse, R. P. J. Med. Chem. 1993, 36, 331

[55] Lee, S. B.; Park, Y. I.; Dong, M. S.; Gong, Y. D. Bioorg. Med. Chem. Lett. 2010, 20, 5900.

[56] Badran, M. M.; Abouzid, K. A. M.; Hussein, M. H. Arch. Pharm. Res. 2002, 26, 107.

[57] Dailey, S.; Feast, W. J.; Peace, R. J.; Sage, I. C.; Till, S.; Wood, E. L. J. Med. Chem. 2001, 11, 2238.

[58] Schneidenbach, D.; Ammermann, S.; Debeaux, M.; Freund, A.; Zollner, M.; Daniliuc, C.; Jones, P. G.; Kowalsky, W.; Johannes, H. H. Inorg. Chem. 2010, 49, 397.

[59] Gao, J.; Ren, Z. G.; Lang, J. P. Chin. Chem. Lett. 2017, 28, 1087.

[60] Guzmán-Percástegui, E.; Hernández, D. J.; Castillo, I. Chem. Commun. 2016, 52, 3111

[61] Monnereau, L.; Sémeril, D.; Matt, D.; Gourlaouen, C. Eur. J. Inorg. Chem. 2017, 2017, 581.

[62] Aloui, L.; Abidi, R.; Chetcuti, M. J. Inorg. Chim. Acta 2020, 505, 119494

(Cheng, F.) 